\title{
Intolerance of Uncertainty as a Transdiagnostic Mechanism of Psychological Difficulties: A Systematic Review of Evidence Pertaining to Causality and Temporal Precedence
}

\author{
Benjamin A. Rosser ${ }^{1}$ (D)
}

Published online: 22 September 2018

(c) The Author(s) 2018

\begin{abstract}
Intolerance of uncertainty (IU) is proposed to be a transdiagnostic causal mechanism of psychological difficulties. The systematic review sought to evaluate the status of evidence pertaining to IU's proposed causal influence upon symptoms of psychological conditions. The review collated evidence from studies involving experimental manipulation and assessment of temporal precedence to ensure direct assessment of causality. The search strategy and eligibility screening identified 12 articles, detailing 15 eligible studies (experimental manipulations: $n=10$; temporal precedence studies: $n=5$ ). Available evidence comprised symptoms of anxiety- and mood-related conditions, including obsessive-compulsive disorder (OCD). The greatest support for IU as a causal mechanism was evident for anxiety-related difficulties and, to a lesser extent, negative affect; limited support was found for OCD-related difficulties. However, notable inconsistency across study findings for all difficulty types precludes absolute conclusions. Implications and recommendations are discussed.
\end{abstract}

Keywords Intolerance of uncertainty $\cdot$ Transdiagnostic $\cdot$ Psychological mechanism $\cdot$ Anxiety $\cdot$ Depression $\cdot$ Obsessivecompulsive disorder

\section{Introduction}

Uncertainty is a pluralistic state in which there is insufficient information to find resolution. The experience of uncertainty can be aversive and threatening. Intolerance of uncertainty (IU) refers to difficulty enduring the experience of not knowing and can give rise to a range of cognitive, emotional, and behavioural responses aimed at avoiding and/or resolving the aversive experience (Carleton 2016). More than a symptom of difficulty, researchers have proposed IU to be a maintaining mechanism and suggested that the range of associated responses may illustrate IU's transdiagnostic relevance to understanding and supporting a breadth of psychological difficulties (e.g., Carleton 2012, 2016; Einstein 2014).

Electronic supplementary material The online version of this article (https://doi.org/10.1007/s10608-018-9964-z) contains supplementary material, which is available to authorized users.

Benjamin A. Rosser

B.A.Rosser@ljmu.ac.uk

1 School of Natural Sciences and Psychology, Liverpool John Moores University, Liverpool L3 3AF, UK
IU is a hallmark of anxiety conditions, demonstrating association with difficulties including generalized anxiety (Ladouceur et al. 1999), social anxiety (Boelen and Reijntjes 2009), panic (Carleton et al. 2014), and obsessive-compulsive difficulties (Holaway et al. 2006; Tolin et al. 2003). ${ }^{1}$ Meta-analysis has demonstrated strong positive correlation between IU and anxiety-related difficulties, as well as higher IU levels in clinical compared to non-clinical populations (Gentes and Ruscio 2011). A recent pathway analysis largely demonstrated association between IU and both conditionspecific vulnerabilities (e.g., generalised anxiety-related 'negative metacognitions') and condition symptoms themselves (Shihata et al. 2017). This research also suggests that, while trait IU may present as a general vulnerability underpinning multiple difficulties, consideration of condition-specific IU may help clarify the differing trajectories associated with this proposed common mechanism. Consistent with the notion of divergent trajectories and multifinality (see Nolen-Hoeksema and Watkins 2011), uncertainty may present as aversive and intolerable for many; however, different

\footnotetext{
${ }^{1}$ Obsessive-compulsive disorder was formally classified as an 'anxiety disorder' within DSM-IV (APA, 2000), but has since been separated from other anxiety difficulties within DSM-5 (APA, 2013).
} 
configurations of concern, interpretation, and response to that experience may be associated with different psychological difficulties. The manifestation of these difficulties may extend beyond anxiety.

Evidence supports the proposition that IU may have relevance to a range of other psychological difficulties. There is substantial correlational evidence to suggest IU is associated with depression (Gentes and Ruscio 2011). Not only have IU levels appeared significantly higher in clinical samples of individuals with depression compared to non-clinical samples, but the distribution of IU levels also appears comparable across depression and anxiety-related conditions, with the possible exception of 'panic disorder' (Carleton et al. 2012). This evidence is consistent with meta-analytic support suggesting that IU may contribute substantially to the core latent process of cognitive vulnerability underpinning difficulties across emotional difficulties (Hong and Cheung 2015).

Outside of anxiety and depression, meta-analysis has also demonstrated elevated levels of IU in individuals with eating disorders compared to controls; leading the authors to propose IU as a potential therapeutic target (Brown et al. 2017). Additionally, although less established, IU may also have relevance to psychosis. In a sample of 27 individual experiencing psychosis, White and Gumley (2010) found positive correlations between IU and a range of distress-related beliefs and responses, such as avoidance, hyper-arousal, and perceived loss of control. Furthermore, worry (a key experience in anxiety difficulties) is proposed to be a core component process contributing to difficulties associated with psychosis (Freeman and Garety 2014), which supports the theoretical basis for considering a potential link between IU and psychosis. In summary, the available evidence supports the proposition that IU may be an underlying transdiagnostic mechanism and vulnerability associated with the presence of a range of psychological difficulties.

Transdiagnostic, mechanism-based conceptions of psychological difficulties are arguably of increasing importance, as both clinical (Awenat et al. 2013) and research communities (Cuthbert and Insel 2013) call for more explanatory alternatives to the hegemonic diagnostic model. The validity and utility of the traditional diagnostic model has increasingly come under question. High levels of comorbidity between conditions challenge the supposed discreteness of psychiatric diagnoses, as well as alluding to potential common underpinning mechanisms (Krueger and Eaton 2015). Developing our understanding of transdiagnostic mechanisms may facilitate the development of more effective, efficient, and unifying interventions and support. However, causal, rather than purely associative, relationships between proposed mechanisms such as IU and condition symptoms require substantiation to warrant intervention development and delivery.
Hierarchical consideration of evidence is necessary to establish whether IU exhibits a causal influence upon condition symptoms. Correlational evidence provides a sensible association-based foundation for supporting a relation between IU and multiple psychological difficulties. Correlational data, however, provide insufficient evidence to conclude a causal relation. Kazdin (2007) highlights the importance of additional forms and targets of assessment, such as: (1) experimental manipulation to demonstrate that change in a mechanism causes change in a symptom, and (2) demonstration of temporal precedence, such that mechanism change precedes symptom change.

Existing systematic review provides evidence of correlational associations between IU and multiple psychological difficulties (Gentes and Ruscio 2011). However, uncertainties remain; extension beyond correlation to direct assessment of causality through experimental and longitudinal designs remains essential to fully understanding IU's contribution to psychological difficulties and informing appropriate intervention (see Shihata et al. 2016 for review). Individual studies employing experimental manipulation and assessment of temporal precedence exist. For example, Mosca et al. (2016) conducted an experimental manipulation based on Grenier and Ladouceur (2004), involving participants progressively considering potential outcomes of a possible negative future life event followed by reading statements designed to induce high or low IU. Mosca et al. (2016) reported significantly higher levels of worry (after controlling for baseline worry levels) in the high IU manipulation condition, compared to the low IU and control conditions. However, the study authors only demonstrated this significant difference in the second of two similar manipulation studies reported within the same article. This discrepancy may be attributable to a larger sample size and slight methodological differences in the manipulation task in the second study, compared to the first; however, similar discrepancy has also been found between studies by independent research teams employing equivalent manipulation procedures (Faleer et al. 2017-study 1; Rosen and Knäuper 2009). The observed discrepancies highlight a need for aggregation of experimental evidence exploring the potential causal influence of IU upon psychological difficulties. This aggregation is currently absent. Similarly, while some have reported that change in IU occurs prior to symptom change (e.g., Bomyea et al. 2015), others have reported less definitive evidence (e.g., Goldman et al. 2007). Consequently, while individual studies involving experimental manipulation of IU and assessment of temporal precedence exist, the discrepancies outlined highlight the need for a comprehensive synthesis and review of this evidence. 


\section{Review Objectives}

The primary review objective was to provide systematic summary and interrogation of the current status of evidence regarding IU as a causal transdiagnostic mechanism of psychological difficulty. The review aimed to build upon existing correlational evidence (Gentes and Ruscio 2011) by evaluating the extant body of research directly assessing causality through data pertaining to experimental manipulation and longitudinal assessment of temporal precedence. As human experience and difficulty may be considered as dimensional (ranging from the general population to clinical populations), rather than a dichotomous separation (Widiger and Samuel 2005), the review was not limited to clinical samples and data pertaining to individual symptoms of psychological conditions in any sample were considered relevant.

\section{Method}

\section{Search}

The systematic review of the literature employed a Boolean search strategy comprising the core search components of 'intolerance of uncertainty' and 'psychological condition'see Supplementary Material. An extensive range of relevant synonyms comprised each core component and 'psychological conditions' were based on the Diagnostic and Statistical Manual of Mental Disorders, 5th edition (DSM-5; American Psychiatric Association 2013). The review adhered to PRISMA guidance for systematic reviews (Liberati et al. 2009); however, a review protocol was not registered.

\section{Study Selection}

Eligibility screening of articles involved three stages: (1) review of titles and abstracts to ascertain initial relevance, (2) review of potentially relevant articles in full, and (3) systematic extraction of data from the relevant qualifying articles. In addition, prior to final extraction, the reference lists of studies identified for final inclusion were also screened for additional articles. The review author completed all stages of screening. A second reviewer performed a blind screening of a random subset $(n=25)$ of articles identified at stage two-randomisation was achieved using Microsoft Excel's random number generator. Reviewers demonstrated $84 \%$ agreement. The reviewers resolved disagreement through discussion without the need for arbitration. Without exception, discussion led to resolution of disagreement in support of the primary reviewer's appraisal.

\section{Information Sources}

The search was conducted in February 2018 through the following electronic databases: Academic Search Complete, CINAHL Plus, Medline, PsycARTICLES, PsycINFO, and Web of Science. The search did not employ date restrictions. Where possible 'human' sample was included as an additional search limiter.

\section{Eligibility Criteria}

\section{Studies Types}

Studies eligible for inclusion involved either experimental manipulation or longitudinal assessment of IU (i.e., temporal precedence studies).

Experimental Manipulation Studies The review objective was to determine the influence of IU, rather than evaluate a specific manipulation technique. Consequently, any successful IU manipulation was eligible for inclusion, irrespective of manipulation technique employed. Successful manipulations constituted those demonstrating statistically significant within-group change in IU pre-to-post manipulation and/or statistically significant difference in IU between experimental groups, post-manipulation. The review excluded unsuccessful manipulations as they could not inform evaluation of the subsequent impact of IU change upon symptoms of psychological difficulty.

Studies involving therapeutic interventions were eligible as manipulation evidence, only if the intervention consisted of one intervention component solely targeting IU as the mechanism of change. Intervention studies involving multiple treatment components and multiple potential process variables could not guarantee the specific agent of change and therefore did not qualify as manipulation evidence.

Temporal Precedence Studies Longitudinal studies were eligible for inclusion if they included assessment of temporal precedence of change in IU in relation to change in symptom level (i.e., involving time-lag analysis). Studies involving assessment of temporal precedence required a minimum of three assessment points to ensure that the reported evidence extended beyond concurrent change between IU and symptom level; studies involving experimental manipulation did not need to meet this requirement due to the direct manipulation of IU.

Studies involving therapeutic interventions were eligible as temporal precedence evidence providing they met the criteria outlined above. As temporal precedence evidence pertains to sequence of change and not IU manipulation, the review did not impose an eligibility criterion in terms of intervention content and therapeutic focus. 


\section{Participants}

The review did not apply eligibility restrictions based on participant characteristics (e.g., age, sex, clinical diagnosis).

\section{Outcomes and Measures}

Eligible studies included assessment of both IU and at least one symptom associated with a psychological condition. The process variable of interest was IU. Primary outcome variables were any symptom (or symptom cluster) relating to a recognised psychological condition based on the DSM taxonomy. The review did not include eligibility restrictions based on specific measures (e.g., particular questionnaires); behavioural, interview, and self-report measurements were all eligible. For all included variables, both validated and unvalidated assessment methods were permitted within the review.

\section{Additional Exclusion Criteria}

Studies were ineligible for inclusion if the report involved:

(a) Cross-sectional non-experimental data only;

(b) Single-case designs;

(c) Retrospectively recalled data only;

(d) Sample size $<12$. Criterion based on power analysis assessment of the minimum number of participants required to power the most basic relevant analysis (i.e., a paired samples $t$ test) to detect a large effect;

(e) Manipulation studies in which:

i. The manipulation (or intervention qualifying as manipulation) was unsuccessful in changing IU;

ii. The intervention did not solely target IU

(f) Longitudinal studies (including interventions) that included fewer than three relevant assessment points

(g) Studies not appearing in a peer-reviewed journal or were poster abstracts;

(h) Studies not reported in English.

\section{Data Collection Process}

Data were systematic extracted using a standardised form created for the purposes of the present review. In the event of ambiguity in study report and/or absence of necessary data for effect size calculations, the review author consulted with study authors and/or supplementary datasets where possible.

\section{Data Items}

Extracted data pertained to sample information, study design, assessment measures, analytic methods, and outcomes. Data extracted were from variables relevant to the review focus only (i.e., IU and symptoms of psychological difficulty). Similarly, extracted outcomes were from analyses relevant to the review focus of assessing the impact of IU manipulation and/or temporal precedence only. Mediation analyses were extracted providing they were not based on purely cross-sectional data and their focus was relevant: i.e., analysis assessed IU mediation between manipulation group and symptoms in experimental manipulation studies; or time-lagged IU mediation between time and symptoms in temporal precedence studies. Extracted data also included those required to calculate effect sizes for magnitude of IU observed within each study.

\section{Summary Measures}

The review aimed to provide narrative synthesis rather than calculate study outcome effect sizes or effect size aggregates. These statistics were not anticipated to be reliably calculable given the necessary range of evidence permitted within the review to enable evaluation of the potential transdiagnostic breadth of IU's influence. However, to facilitate interpretation of results, the review did aim to provide evidence of the magnitude of IU level observed within individual studies. Review Manager (RevMan 2014, version 5.3) was used to calculate effect sizes (Hedges' adjusted $g$ ), 95\% confidence intervals, and produce forest plots. The included effect sizes comprised standardised difference in mean IU level between-groups (e.g., high vs. low IU) for the manipulation studies; and standardised within-group change in mean IU level (first to last assessment point) for the temporal precedence studies. This approach provided a pragmatic way to assess magnitude of IU consistently across studies based on typically available data; however, limitations remain. Between-group difference is not a measure of change, and reducing within-group change to two time-points does not necessarily capture the largest change in IU across the measured timeframe. Hence, the included statistics provide a tentative indication of the magnitude of IU difference or change within individual studies to support the narrative synthesis.

\section{Risk of Bias: Quality Assessment}

Study quality was assessed with reference to the Cochrane risk of bias tool (see Higgins and Green 2011). A narrative, rather than numerical, assessment of quality was preferred as it provided greater transparency of assessment. 
Fig. 1 Flow diagram of study eligibility screening and selection

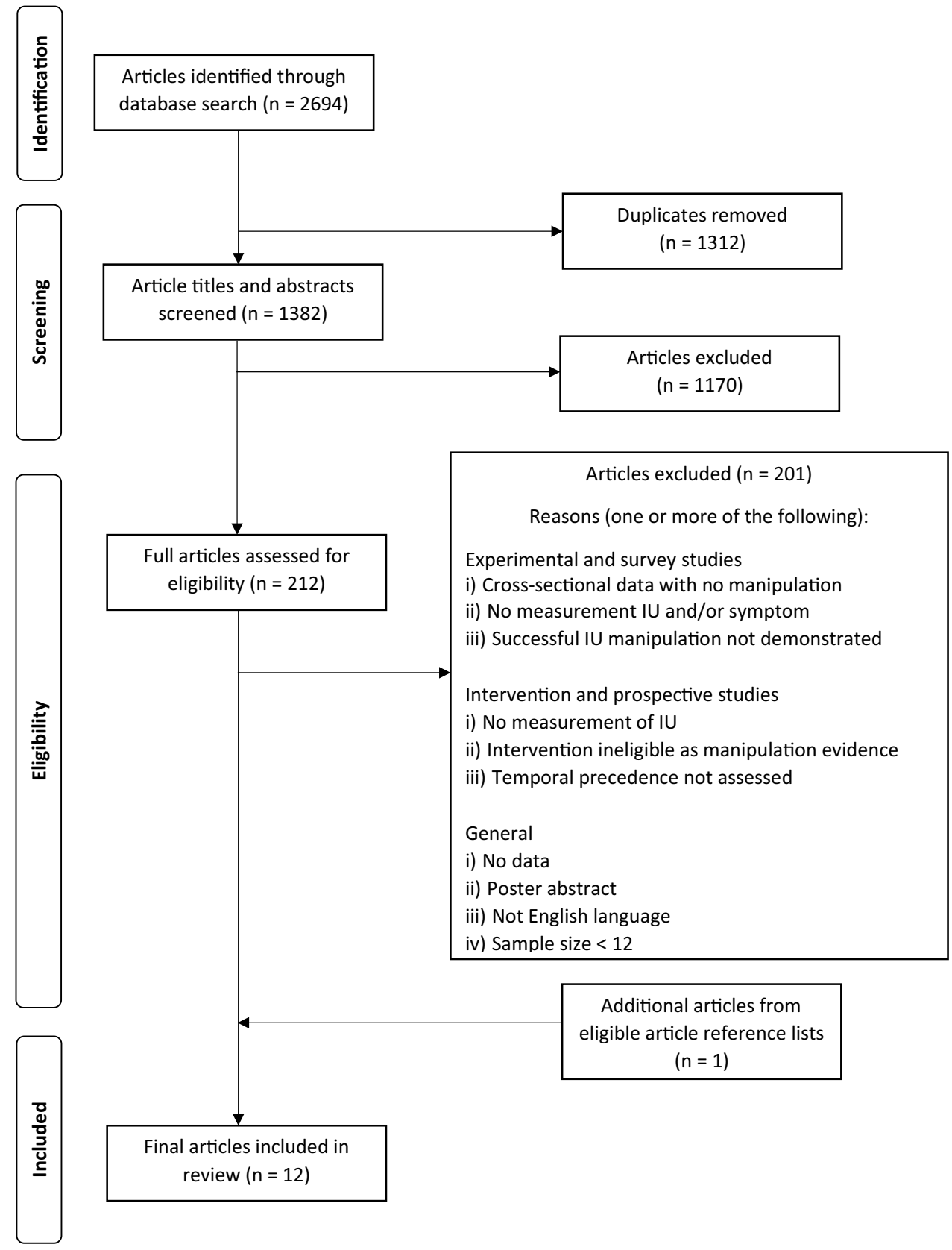

\section{Results}

\section{Study Selection}

The initial database search identified 2694 articles. Following removal of duplicates and review of titles and abstracts, the search identified 212 articles for full screening. Eligibility criteria were met by 11 articles. Common reasons for exclusion were that studies reported cross-sectional data without IU manipulation or lacked assessment of temporal precedence. A search of the reference lists of the 11 identified articles revealed one additional article for inclusion. At completion, the outlined screening procedure identified 12 articles (presenting 15 eligible studies) fulfilling the specified criteria for inclusion in the final review-see Fig. 1.

\section{Study Characteristics}

Study characteristics are summarised in Table 1 . The studies were conducted in a variety of countries, with the USA being the most frequent location $(n=6)$. In all cases, studies reported adult samples. Samples comprised more females than males in all but two studies (Su et al. 2016; Wilhelm et al. 2015). Where articles reported ethnicity data, white/ Caucasian was the most frequently endorsed category. All articles presented data from distinct cohorts. Two articles 


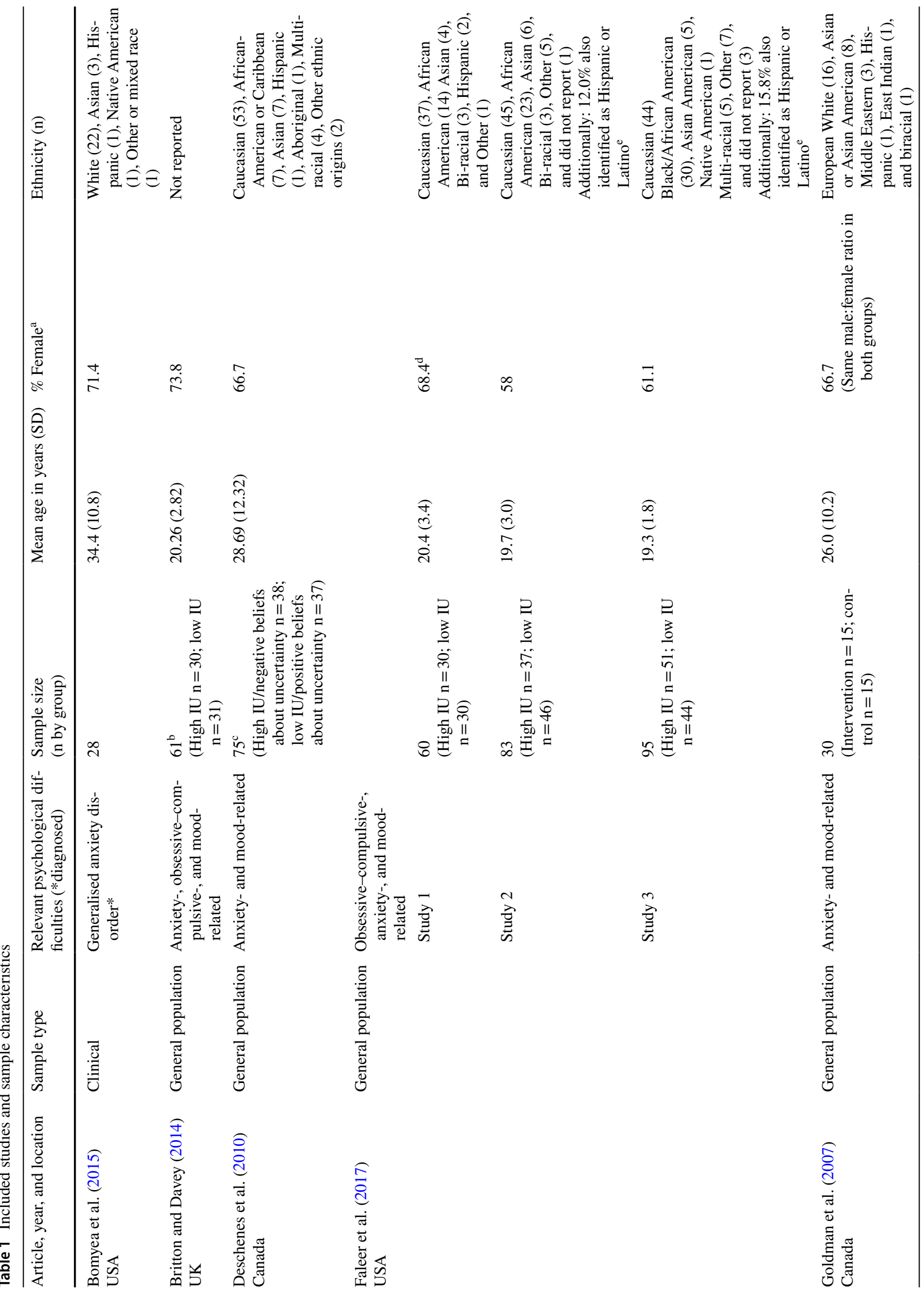




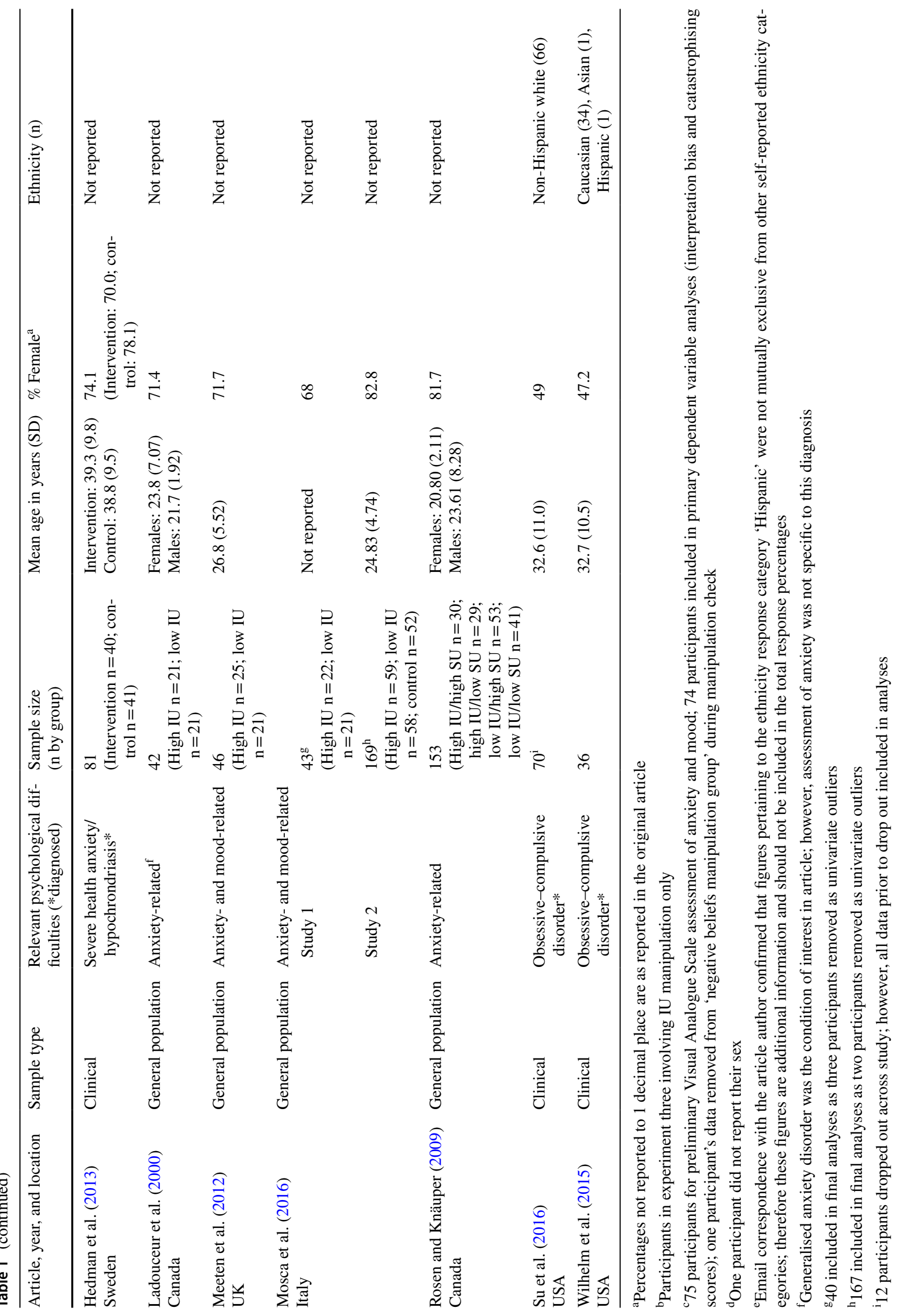


presented eligible data from multiple studies, contributing five studies between them (Faleer et al. 2017; Mosca et al. 2016).

The psychological difficulties focused on within the studies were predominantly anxiety- and mood-related. Only four studies involved clinical samples; focusing on generalised anxiety disorder (GAD) (Bomyea et al. 2015), hypochondriasis (Hedman et al. 2013), and obsessive-compulsive disorder (OCD) (Su et al. 2016; Wilhelm et al. 2015). All other studies involved non-clinical samples.

\section{Study Designs}

Included studies investigated the influence of IU through experimental manipulation or temporal precedence as a process variable of a therapeutic intervention. Experimental manipulations accounted for ten of the included studies (Britton and Davey 2014-study 3 included in review only; Deschenes et al. 2010; Faleer et al. 2017-studies 1-3; Ladouceur et al. 2000; Meeten et al. 2012; Mosca et al. 2016-studies 1 \& 2; Rosen and Knäuper 2009). Intervention studies involving assessment of temporal precedence of IU change in relation to symptom change featured in five articles (Bomyea et al. 2015; Goldman et al. 2007; Hedman et al. 2013, Su et al. 2016; Wilhelm et al. 2015).

Manipulations All experimental studies manipulated participants into either high or low IU groups for comparison. Only one experimental study involved a non-manipulated control comparison (Mosca et al. 2016-study 2). Deschenes et al. (2010) manipulated participant beliefs about uncertainty to be either negative or positive. The authors characterised IU as negative beliefs about uncertainty and demonstrated significantly different levels of IU between groups. Consequently, the review presents the results from this study as comparable to the other high/low IU manipulations.

Manipulation procedures varied across studies. Two studies utilised identical manipulation procedures (Faleer et al. 2017; Rosen and Knäuper 2009). The linguistic manipulation employed (based on Salancik and Conway 1975) involved altering the wording of IU questionnaire response options to increase or decrease the likelihood of statement endorsement, followed by rigged standardised feedback about the participant's ability to tolerate uncertainty, dependent on manipulation group. A discrepancy between these two studies, however, was that Rosen and Knäuper (2009) also employed a manipulation of 'situational uncertainty' through information provision that either did, or did not, encourage ambiguity as to the likelihood of participants having a fictitious health condition. The study considered both main effects and the interaction between these two manipulations.
The two studies presented in Mosca et al. (2016) employed near identical IU manipulations involving a Vertical Arrow Technique (VAT). This manipulation replicated Grenier and Ladouceur (2004) with the modification of focusing on a personal negative, rather than neutral, possible future life event. During the VAT procedure, participants reported potential sequential consequences stemming from an initial possible negative life event. The procedure was similar to that of the Catastrophising Interview (see "Assessment Methods and Outcomes" below), but was self-administered. High and low IU manipulation was initiated through participants subsequently reading statements relating to an inability or ability to tolerate uncertainty, respectively. The manipulation procedure was slightly adapted between study one and two, largely in terms of: (1) an increase in delay between VAT and statements (this interval was included as a covariate in subsequent analysis), (2) refreshing memory of VAT, and (3) ceasing to direct participants to read the manipulation statements aloud.

The preferred method of manipulation in two studies was through a vignette, which described a protagonist experiencing either high or low IU. Follow-up instructions differed between these studies, such that participants either provided advice for the vignette protagonist (Britton and Davey 2014) or considered a personalised uncertain situation from the protagonist's perspective (Meeten et al. 2012).

The remaining two studies reported relatively standalone manipulation techniques. Deschenes et al.'s (2010) manipulation involved a Powerpoint presentation on the impact of uncertainty on problem solving, emphasising the impact as negative or positive dependent on manipulation group. Ladouceur et al. (2000) employed a rigged-outcome gambling task, emphasising or minimising the likelihood of negative outcome and consequence dependent on manipulation group.

All manipulation studies included a manipulation check. Post-manipulation high/low IU group differences provided evidence of successful manipulation in five studies (Britton and Davey 2014; Deschenes et al. 2010; Faleer et al. 2017—study 1; Ladouceur et al. 2000; Meeten et al. 2012). A further five studies reported post-manipulation group differences after controlling for pre-manipulation IU scores (Faleer et al. 2017-studies 2 \& 3; Mosca et al. 2016-studies $1 \&$ 2; Rosen and Knäuper 2009). Additionally, Mosca and colleagues introduced a control group in study two and reported that the group differences were evident in all group comparisons, with the exception of low IU compared to control group. Finally, three studies assessed within-group increase and decrease in high and low IU groups, respectively (Faleer et al. 2017—studies 2 \& 3; Rosen and Knäuper 2009). Each of these studies demonstrated evidence of significant IU change pre-to-post manipulation in the intended direction, with the exception of Faleer et al. (2017—study 
3) who found significant change in the low IU group only. The study authors concluded that manipulation was only partially successful.

Interventions. The review did not identify any intervention studies with sufficient specificity in terms of therapeutic focus and agent of change to meet eligible criteria for inclusion as manipulation evidence.

Temporal Precedence Evidence pertaining to temporal precedence included studies incorporating assessment of time-lagged mediation or prediction models. In this review, this type of evidence came exclusively from intervention studies; no non-intervention prospective studies were identified.

Of the studies evaluating temporal precedence, two studies conducted eligible mediation analyses investigating time-lagged IU level as a mediator of symptom level across time (Bomyea et al. 2015, Su et al. 2016). Whilst Hedman et al. (2013) included both traditional mediation analysis and time-lag analysis of mediator on symptoms, only the latter was eligible for inclusion in this review. The remaining two studies did not conduct a full traditional mediation analysis, but did assess time-lagged IU level (Goldman et al. 2007) or time-lagged change in IU (Wilhelm et al. 2015) as a predictor of subsequent symptom level.

There were additional variations in analytic approach between studies. IU at the same time point as the symptom outcome was additionally controlled for in one study (Goldman et al. 2007); and two studies controlled for symptom level at the previous time point (Hedman et al. 2013, Su et al. 2016). Su et al. (2016) also included depression within their mediation analysis as a non-specific mediator. The remaining two studies included no additional covariates (Bomyea et al. 2015; Wilhelm et al. 2015).

Interventions. Cognitive and behavioural models of psychological difficulties typically informed the included interventions. Consequently, interventions aimed to target and test the accuracy of difficult cognitions and/or increased contact with difficult experiences through exposure, but not always in combination.

Interventions included cognitive behavioural therapy (CBT) delivered as an internet-based intervention (Hedman et al. 2013) and as a computer-assisted face-to-face intervention (Bomyea et al. 2015). Hedman et al. (2013) reported a 12-week intervention based on the cognitive behavioural model, with the primary focus being exposure and response prevention $(\mathrm{EX} / \mathrm{RP})$. The intervention also included mindfulness training as a form of exposure work. Bomyea et al. (2015) reported focus on exposure as well as cognitive restructuring in their ten-session intervention. Both interventions utilised modular treatment formats and incorporated elements such as psychoeducation and relapse prevention. Neither intervention included a treatment component specifically targeting IU, but both considered IU a potential intervention process variable based on cognitive behavioural theory.

Wilhelm et al. (2015) reported a 22-session cognitive therapy intervention primarily focusing on cognitive change. The behavioural components included in the intervention were in the form of behavioural experiments designed to test cognitions, rather than progressive exposure work.

The remaining two studies employed solely exposurebased interventions in the form of EX/RP ( $S u$ et al. 2016) and written exposure (Goldman et al. 2007). The EX/RP intervention was therapist delivered and consisted of 17 twice-weekly sessions in the acute treatment phase-during the subsequent maintenance phase additional full sessions were also available as required to meet a standardised symptom threshold, before transitioning to half-length sessions. The written exposure intervention consisted of five sessions in which participants described the same 'worst fear coming true' and were encouraged to include increasing depth of description across sessions. The control condition in this study followed the same instructions for an unemotional possible future event. Of the five studies, only two included control conditions for comparison (Goldman et al. 2007; Hedman et al. 2013); however, the latter study appeared to collapse treatment and control conditions for the temporal precedence analysis included in this review.

\section{Assessment Methods and Outcomes}

Intolerance of Uncertainty: Mechanism Measures Assessment of intolerance of uncertainty was, near exclusively, through self-report. The most frequently employed measure was the Intolerance of Uncertainty Scale (IUS; Freeston et al. 1994), also utilised in short-form (IUS-12; Carleton et al. 2007). The full unmodified 27 -item IUS was employed in one study (Goldman et al. 2007); and combined with the 'Predictability of Future Contexts' subscale of the Need for Closure Scale (Webster and Kruglanski 1994) in a further two studies (Faleer et al. 2017-study 1; Rosen and Knäuper 2009). The latter two studies modified questionnaire response options as part of the manipulation task. The shortform IUS-12 was utilised across one intervention study (Bomyea et al. 2015) and was included in three experimental studies for baseline assessment only (Meeten et al. 2012; Mosca et al. 2016-studies $1 \&$ 2). As their primary IU outcome measures, Meeten and colleagues employed a Visual Analogue Scale (VAS), whereas Mosca and colleagues employed the IU subscale of the Worry and Intolerance of Uncertainty Questionnaire (Grenier and Ladouceur 2004). A further three studies utilised five to six IUS items, with (Ladouceur et al. 2000) or without (Britton and Davey 2014; Deschenes et al. 2010) minor adaptation of item phrasing to increase their relevance to the manipulation task. Addi- 
tionally, Deschenes et al. (2010) embedded the IUS items within a personality measure to reduce demand characteristics. Furthermore, this study included the only behavioural assessment of IU using the Probabilistic Inference Task (Garety et al. 1991; Ladouceur et al. 1997), accompanied by a VAS assessment of participant certainty of the accuracy of their task response. However, these assessment methods failed to demonstrate significant difference between manipulation groups, whereas the IUS scores did.

Of the studies involving clinical samples, two studies (Su et al. 2016; Wilhelm et al. 2015) employed the Perfectionism/IU subscale of the Obsessive Beliefs Questionnaire (Obsessive-Compulsive Cognitions Working Group; OCCWG 2005). Consequently, this variable was not solely a measure of IU. Faleer et al. (2017—study 2) utilised the IU subscale from the original 87-item version of same measure (OCCWG 2001, 2003). Within the same paper, the research team also utilised the Intolerance of Uncertainty IndexPart A (Gosselin et al. 2008) as a baseline assessment of IU (study 1) and as an assessment of IU change pre/post manipulation (study 3 ).

Finally, Hedman et al. (2013) utilised the 'Psychological Reactions to Bodily Sensations' subscale of the short version of the Health Anxiety Inventory (Salkovskis et al. 2002). The authors acknowledged that this measure was not an established assessment of IU and urged caution in interpreting results.

\section{Symptoms of Psychological Difficulties: Outcome Meas-}

ures Included studies predominantly explored anxiety- and mood-related difficulties as outcome variables. Assessment of obsessive-compulsive related difficulties also featured, but to a lesser extent.

Anxiety. Assessment of anxiety-related difficulties (excluding OCD-specific symptoms) featured in 11 studies. Assessed difficulties included symptoms of general and condition-specific worry (e.g., health anxiety; GAD somatic symptoms), catastrophising, and interpretation bias.

In terms of non-condition specific worry, the most commonly employed standardised measure was the Penn State Worry Questionnaire (PSWQ; Meyer et al. 1990), which was employed in three studies in either original (Rosen and Knäuper 2009), weekly assessment (Goldman et al. 2007), or abbreviated form (Bomyea et al. 2015). Additionally, one study included baseline PSWQ assessment as a covariate, rather than outcome measure (Faleer et al. 2017-study 1), and one study employed three PSWQ items that had been adapted to reference the manipulation task (Ladouceur et al. 2000). Other validated questionnaires assessing anxiety and/or worry included: Worry and Intolerance of Uncertainty Questionnaire: Worry subscale (Grenier and Ladouceur 2004) in two studies (Mosca et al. 2016 — study 1 \& 2), and State-Trait Anxiety Scale (STAI;
Spielberger 1983) in one study (Rosen and Knäuper 2009). Additionally, anxiety was included as a VAS item within four studies (Britton and Davey 2014; Deschenes et al. 2010; Meeten et al. 2012; Mosca et al. 2016—study 2).

Condition-specific worry was assessed for GAD (Goldman et al. 2007) and health anxiety (Hedman et al. 2013) using the Worry and Anxiety Questionnaire (Dugas et al. 2001) and Health Anxiety Inventory-short version (SHAI; Salkovskis et al. 2002), respectively. In the latter study, the outcome variable was a composite of SHAI 'Disease Conviction' and 'Fear and Worry about Illness' subscales. Additionally, Rosen and Knäuper (2009) included a single item likert-scale assessment of worry due to uncertainty relating to the fictitious health condition involved in the manipulation task.

Three studies employed the researcher-delivered Catastrophising Interview (Deschenes et al. 2010; Faleer et al. 2017-study 1; Meeten et al. 2012). The Catastrophising Interview (Davey 2006; Vasey and Borkovec 1992) employed a VAT to facilitate participant consideration of a personal fear and sequence of potential subsequent consequences. Repetition of the process of consequence generation continued until no further consequences were forthcoming. The number of times the process was repeated to achieve this end was referred to as the number of catastrophising steps. The number of steps was the primary outcome for this assessment measure in Faleer et al. (2017—study 1) and Meeten et al. (2012); whereas Deschenes et al. (2010) also assessed perceived likelihood of consequence occurrence through participant self-report and perceived severity of the final step through researcher evaluation.

Finally, Deschenes et al. (2010) assessed anxiety-related interpretation bias using the Ambiguous/Unambiguous Situations Diary (AUSD; Davey et al. 1992; Koerner and Dugas 2008). The researchers modified the task to include ambiguous scenarios only. Participants rated ambiguous scenarios in terms of worry induced prior to the resolution of the scenario, which was resolved with either positive/neutral or negative outcome. Participants then rated both the likelihood of the outcome and its perceived level of positivity or negativity.

Mood, Affect, and Depression. Assessment of moodrelated difficulties featured in eight studies (Britton and Davey 2014; Deschenes et al. 2010; Faleer et al. 2017studies 1 \& 2; Goldman et al. 2007; Meeten et al. 2012; Mosca et al. 2016-studies $1 \&$ 2). Assessment was via VAS, with the exceptions of utilisation of the Centre for Epidemiological Studies Depression Scale (Radloff 1977) by Goldman et al. (2007); and the Positive and Negative Affect Schedule (PANAS; Watson et al. 1988) by Faleer et al. (2017—study $1 \& 2$; negative affect measured only) and Mosca et al. (2016-study 1). Whilst Su et al. (2016) included the Hamilton Depression Scale (Hamilton 1960) 
and included the depression score as a covariate (rather than outcome measure) in the IU relevant analysis.

Obsessions and Compulsions. Assessment of symptoms relevant to obsessive-compulsive related difficulties featured in six studies. Both Su et al. (2016) and Wilhelm et al. (2015) employed the Yale-Brown Obsessive Compulsive Scale (Y-BOCS; Goodman et al. 1989a, b). Additionally, Faleer et al. (2017—studies 1-3) assessed obsessive-compulsive difficulties using: (1) self-report through the Obsessive Beliefs Questionnaire: Threat Estimation subscale (OCCWG 2001, 2003) and a single item assessment of perceived threat of intrusive thoughts (Clark et al. 2000); and (2) behavioural assessment of checking behaviour-replicating MacDonald and Davey (2005), and Rotge et al. (2008). Finally, a single study assessed inflated responsibility (Britton and Davey 2014) via four items from the Responsibility Attitude Scale (RAS; Salkovskis et al. 2000). In support of this assessment, the authors reported significant positive correlation between the reduced item set and the full RAS. Inflated responsibility may be considered a symptom that is arguably relevant to GAD and depression, as well as OCD (see Britton and Davey 2014).

\section{Risk of Bias Within Studies: Quality Assessment}

\section{Selection Bias}

All but two studies involving multiple participant groups reported randomised group allocation, although frequently studies did not detail the randomisation procedure. The two exceptions either failed to state randomisation (Faleer et al. 2017-study 3) or employed alternating allocation (Mosca et al. 2016-study 1), respectively. The majority of multiple group studies reported statistically comparing groups on some baseline metrics (except Hedman et al. 2013; Ladouceur et al. 2000). Of these studies, six found evidence of baseline differences in IU between groups (Faleer et al. 2017-studies 1-3; Goldman et al. 2007; Meeten et al. 2012; Rosen and Knäuper 2009). Rosen and Knäuper (2009) and Faleer et al. (2017) consequently included the identified baseline variables as covariates in their primary analyses. No studies reported baseline between-group differences on primary outcome measures, with the exception of higher positive affect in the high compared to low IU group in Mosca et al. (2016). Additionally, although not the primary outcome variable, Faleer et al. (2017—study 1) included baseline PSWQ assessed worry as a covariate in subsequent analyses after identifying baseline group differences on this measure.

\section{Performance and Detection Bias}

In terms of participant blinding, six studies explicitly reported concealing the study purpose from participants (Britton and Davey 2014; Deschenes et al. 2010; Ladouceur et al. 2000; Meeten et al. 2012; Mosca et al. 2016-studies 1 \& 2). In addition, one study reported blinding the researcher to participant group (Deschenes et al. 2010) and three studies reported independent or blinded assessors (Deschenes et al. 2010; Hedman et al. 2013, Su et al. 2016). However, this information was only determined for Hedman et al. (2013) after consulting a sister publication detailing the main findings of the full intervention trial.

\section{Attrition Bias}

There was no report of participant dropouts or exclusions in six studies (Bomyea et al. 2015; Britton and Davey 2014; Goldman et al. 2007; Hedman et al. 2013; Ladouceur et al. 2000; Meeten et al. 2012). Of the remaining studies, numbers of participants excluded were typically small and conducted for methodological reasons, although two intervention studies did not report participant reasons for withdrawing (Su et al. 2016; Wilhelm et al. 2015). A considerable number of exclusions $(n=67)$ was reported in one study due to criteria needed to demonstrate successful manipulation (Rosen and Knäuper 2009); however, no statistical difference in age or sex between included and excluded participants was evident.

Missing data were treated using intention-to-treat approaches in two studies (Bomyea et al. 2015; Wilhelm et al. 2015) —with mean replacement where $<10 \%$ of items were missing, in the latter study. In addition, Hedman et al. (2013) statistically substantiated the randomness of missing data, and Su et al. (2016) statistically substantiated no significant influence of dropouts on outcome.

\section{Reporting Bias}

Of the reviewed articles, three were associated with registered trials (Bomyea et al. 2015; Hedman et al. 2013, Su et al. 2016). However, the reviewed articles were not the primary report of those trials. In each case, the registered trial details did not include explicit mention of IU, suggesting that IU was not the immediate focus of the original research. However, overall there was theoretical justification for focus on IU throughout all included studies. Furthermore, both significant and nonsignificant findings were evident, suggesting transparency in report. 


\section{Results of Individual Studies}

\section{Study Outcomes Within Individual Studies}

The summary tables of individual study results provide an overview of analyses and outcomes for both experimental manipulation (Table 2) and temporal precedence studies (Table 3).

The review did not perform a statistical summary of outcome variables due to the heterogeneity of study outcome measures and methods.

\section{Magnitude of IU Within Individual Studies}

The magnitude of IU effect size (Hedges' adjusted $g$ ) was calculated within each study, except for Wilhelm et al. (2015) where necessary data were not available. The effect sizes represent either the difference in IU level between manipulation groups for experimental studies (see Fig. 2) or change in IU level between first and last assessment for temporal precedence studies (see Fig. 3).

Provision of these effect sizes and confidence intervals demonstrates the range and reliability of IU level observed. The data are a tentative guide aimed to facilitate interpretation of the individual study results and comparison across studies. These statistics are not an assessment of overall IU effect or evaluation of the effectiveness of a specific methodology (e.g., a particular manipulation technique). In addition, the review excluded unsuccessful manipulation studies because they lacked significant IU difference/change and therefore could not provide assessment of the subsequent impact of IU difference/change. Consequently, based on the review focus and associated eligibility criteria, tests of publication bias and overall effect size robustness were inappropriate for these data.

\section{Synthesis of Results}

The review studies demonstrated heterogeneity, suggesting meta-analyses of study outcomes would be unwise. Consequently, in the interests of transparency and accurate representation, the review focused on narrative synthesis. The following synthesis stratifies discussion of study results by psychological difficulty and evidence type (namely, experimental manipulation and temporal precedence studies, respectively).

\section{Anxiety}

Experimental Manipulation Studies Investigation of the affect of IU manipulation on anxiety-related symptoms featured in eight experimental manipulation studies-see Table 2. Of the three studies utilising validated multi-item measures of anxiety or worry, two demonstrated no significant difference between manipulations groups, after controlling for baseline anxiety/worry (Mosca et al. 2016-study 1; Rosen and Knäuper 2009). Contrastingly, the remaining one study (Mosca et al. 2016-study 2) reported significantly higher post-manipulation anxiety levels in the high compared to both low IU and control group. Mosca et al. (2016) employed the same questionnaire and analytic strategy across both reported studies; consequently, the different findings may be due to slight modifications in manipulation procedures (such as increasing the interval between pre/ post-manipulation and assessment) and/or increased sample size in study two.

Several additional studies supported Mosca et al. (2016study 2), also demonstrating greater anxiety in the high compared to low IU manipulation group post-manipulation. However, it should born in mind that, rather than using the full validated measure (or subscale), these studies utilised small, modified selections of validated measure items (Ladouceur et al. 2000) or individual item VAS assessment (Britton and Davey 2014; Meeten et al. 2012). These analyses assessed post-manipulation anxiety levels, rather than pre-to-post manipulation change. The two analyses assessing change in anxiety between groups, across time, were both non-significant (Deschenes et al. 2010; Meeten et al. 2012). Finally, Rosen and Knäuper (2009) assessed worry due to uncertainty relating to the fictitious health condition involved in the manipulation task, after controlling baseline anxiety, IU, and motivation to reduce uncertainty. However, the number of (and likely overlap between) covariates, coupled with significant IU group differences only being present in interaction with the situation uncertainty manipulation (outlined previously), limits the weight that can be placed upon this result within the present review.

In addition to anxiety or worry in general, three studies assessed the specific process of catastrophising. Both Faleer et al. (2017—study 1) and Meeten et al. (2012) reported a significantly greater number of average catastrophising steps in the high compared to the low IU group, even after controlling for baseline anxiety (as well as participant sex and baseline IU in the former study). However, Deschenes et al. (2010) reported no significant between-group differences in catastrophising steps or the researcher-rated severity of the final step, although the negative beliefs about uncertainty group did rate these steps as more likely to occur than the positive beliefs group. The differing manipulation methods complicate comparison between studies; however, both articles reporting significant differences achieved larger IU effect sizes between manipulation groups (post-manipulation), compared to Deschenes et al. (2010)—see Fig. 2. Consequently, the strength of manipulation may have had some 


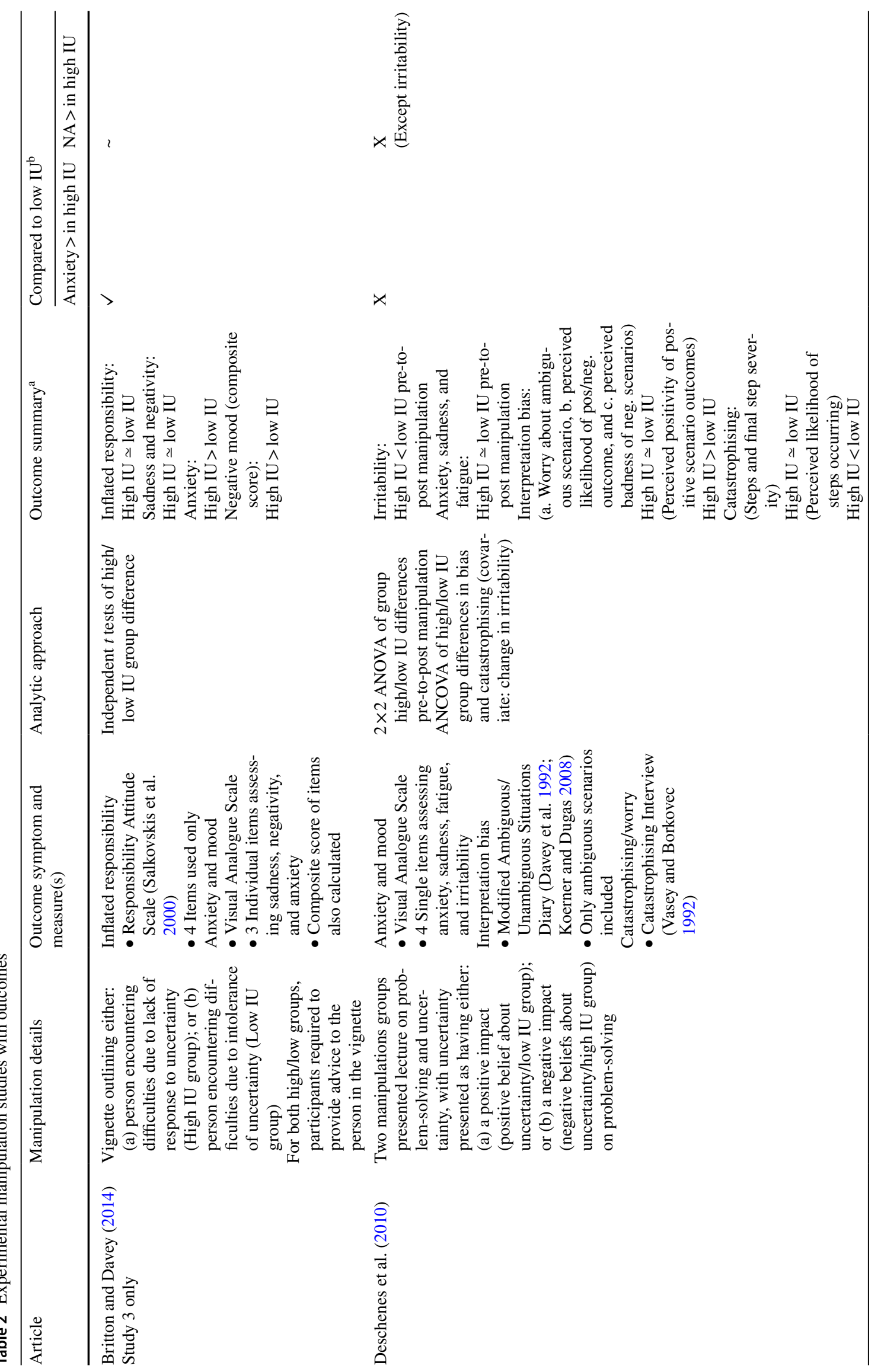




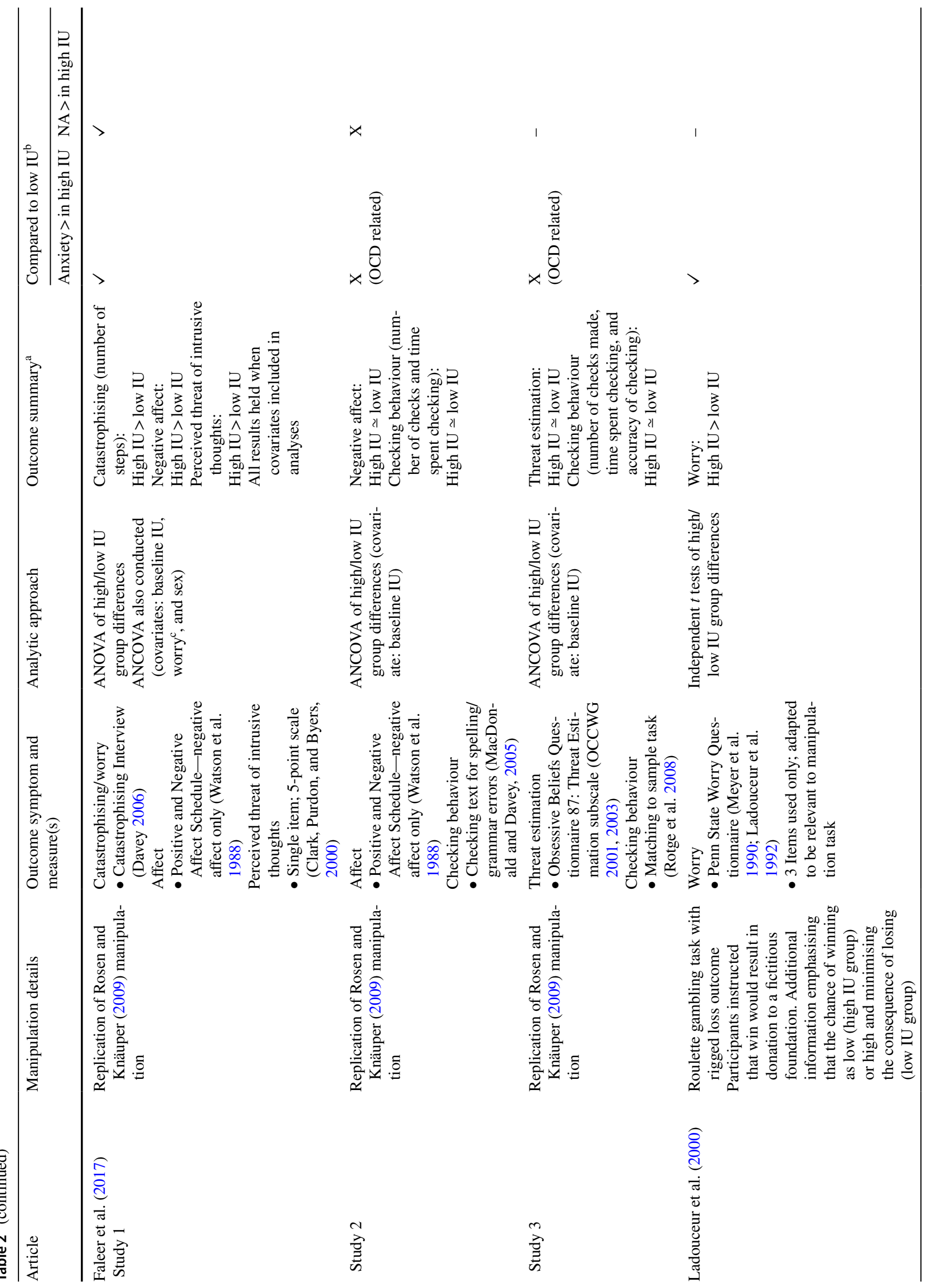




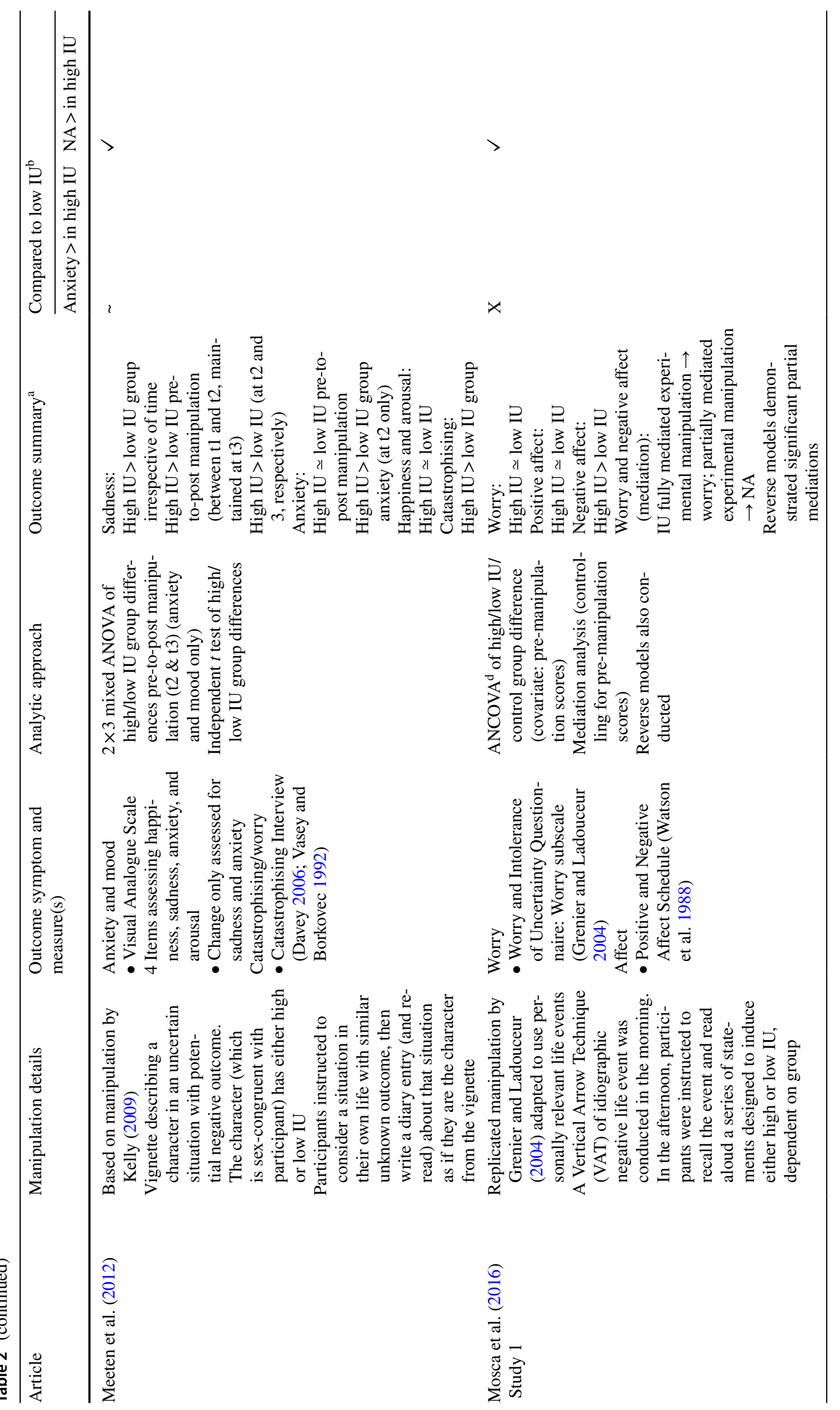




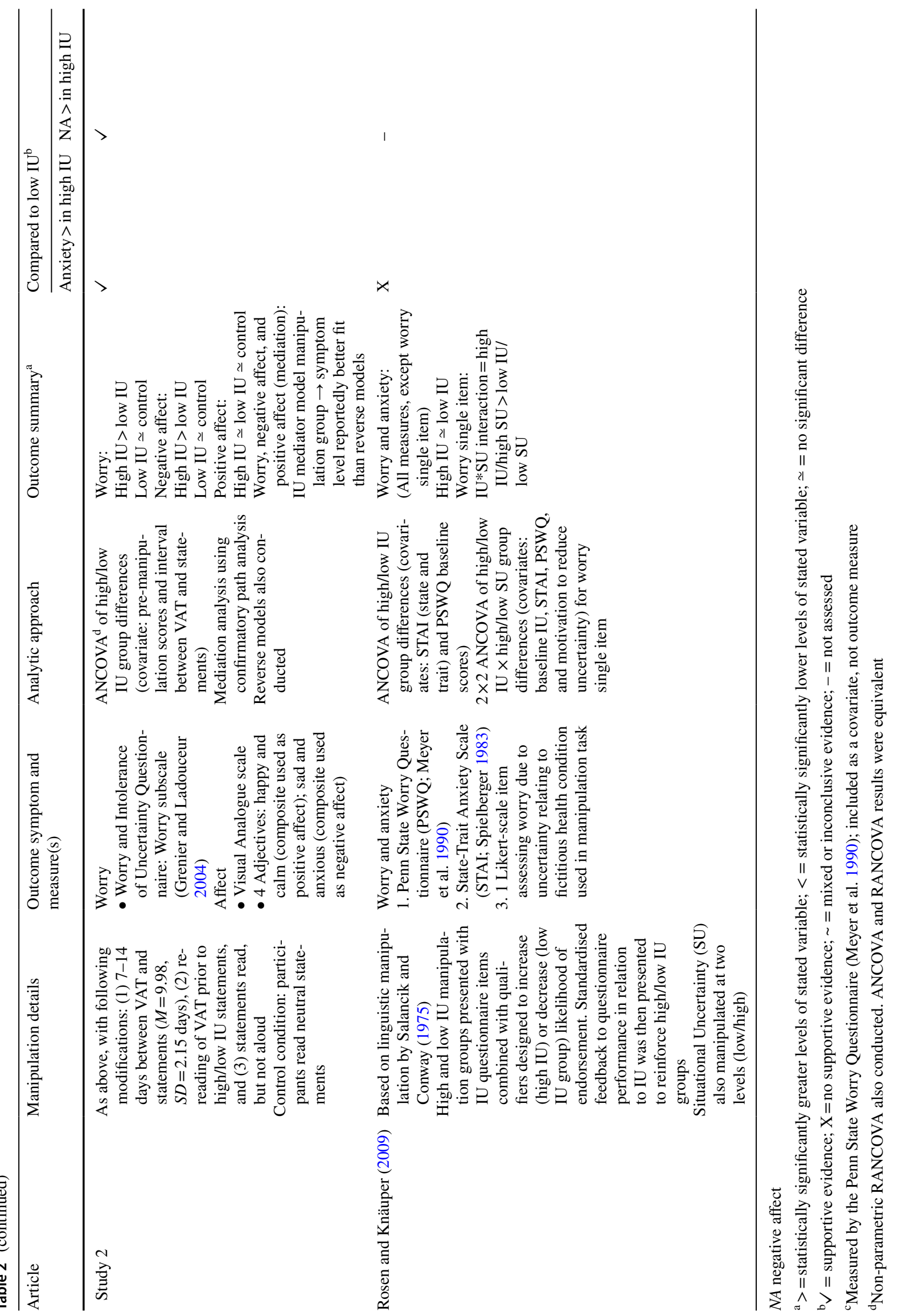




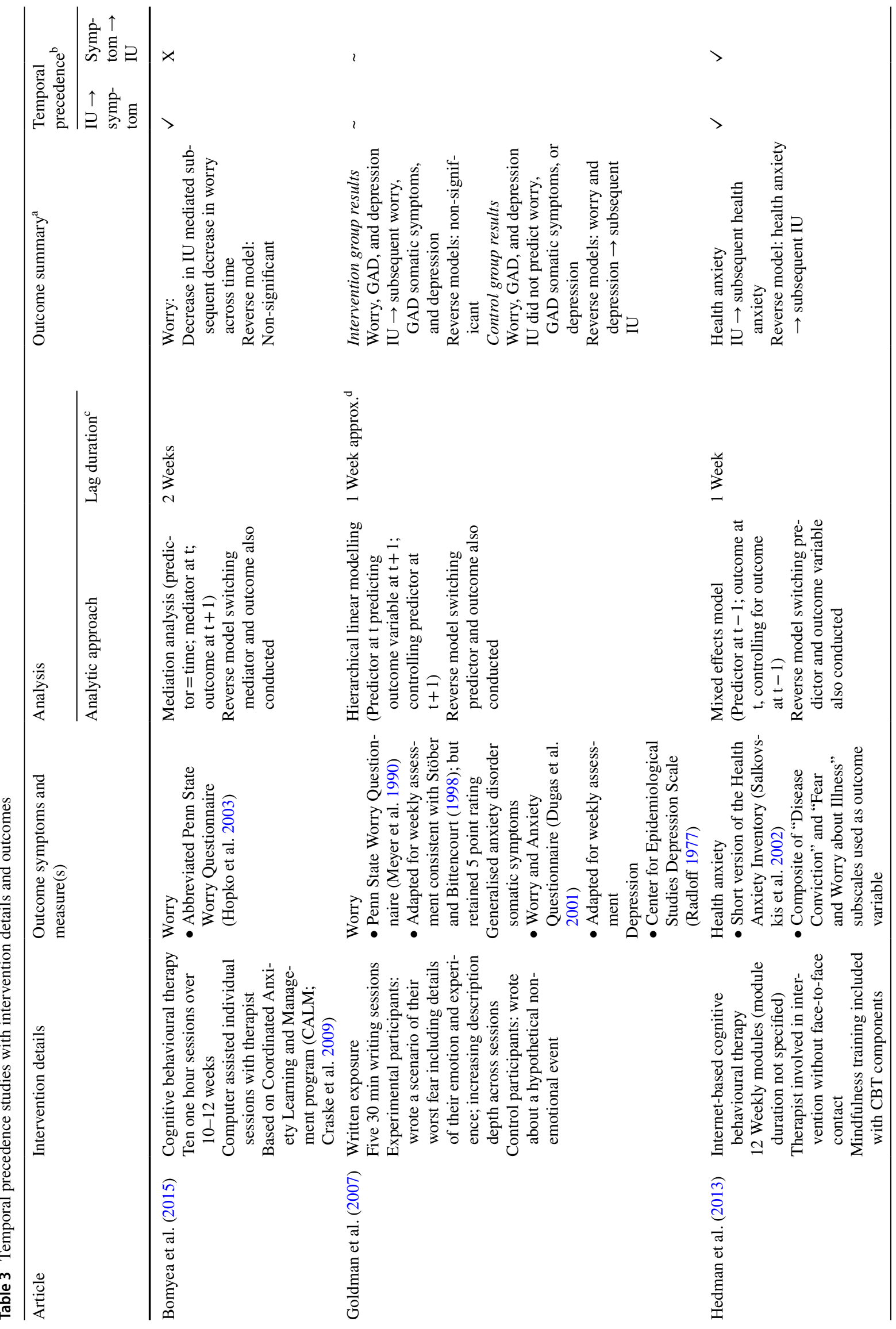




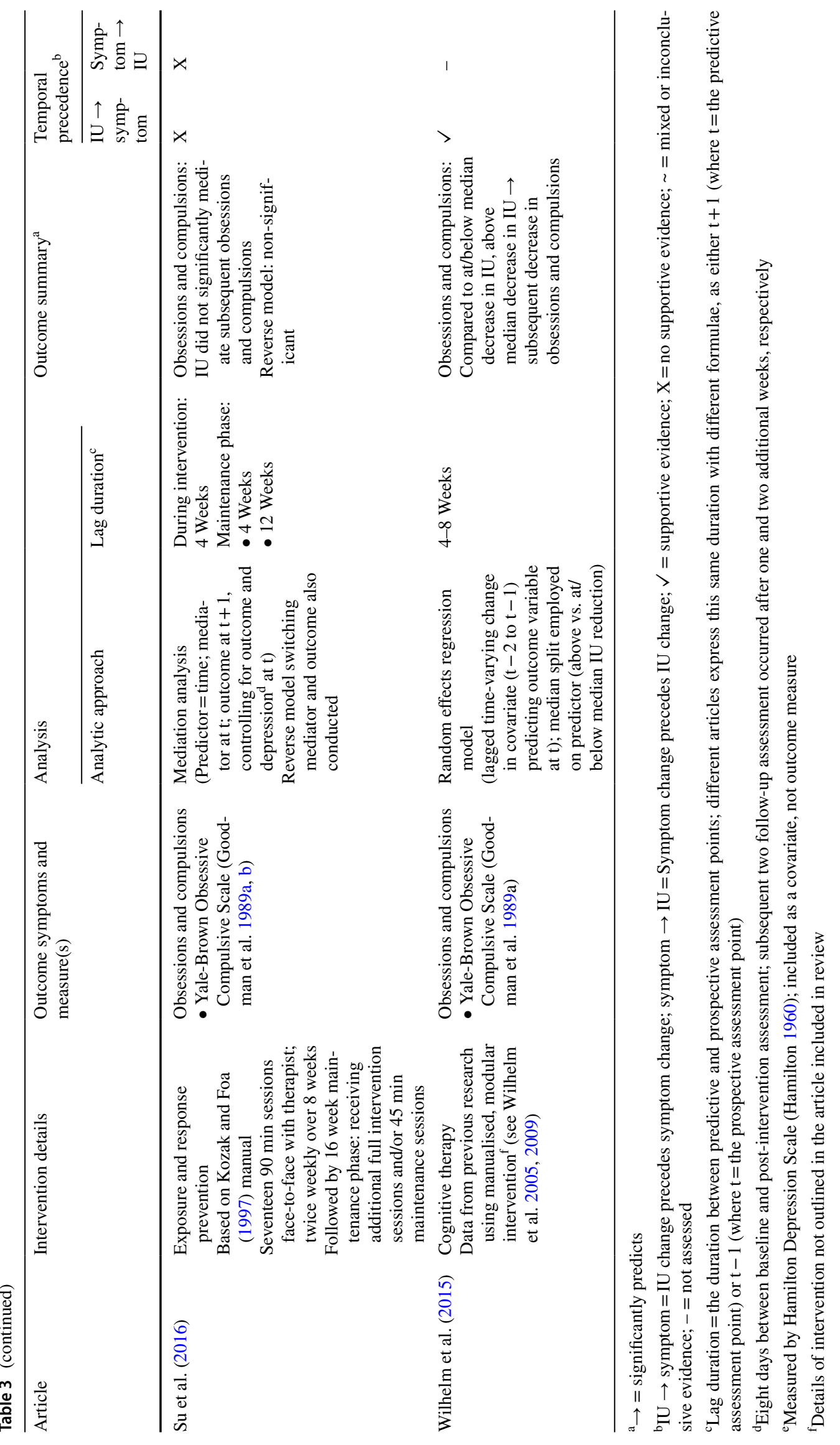


Fig. 2 Forest plot of IU level within individual experimental manipulation studies: Magnitude of difference in IU level (Hedges' adjusted $g$ ) between high and low IU manipulation groups (post-manipulation). additional group comparisons: High IU vs. control group: Hedges' adjusted $g=0.62[0.23$, 1.0]; Low IU vs. control group: Hedges' adjusted $g=0.26$ $[-0.64$ to 0.12$]$

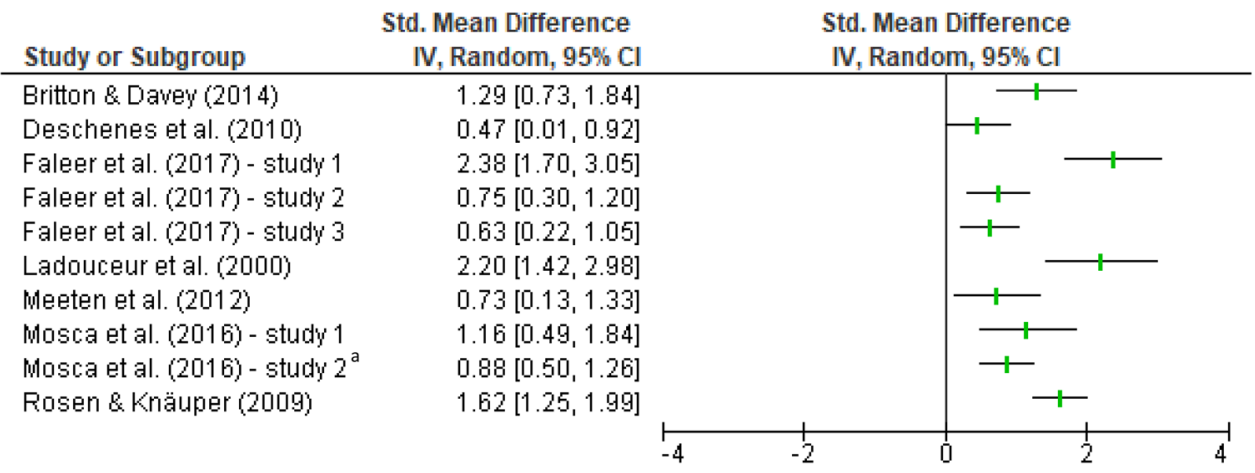

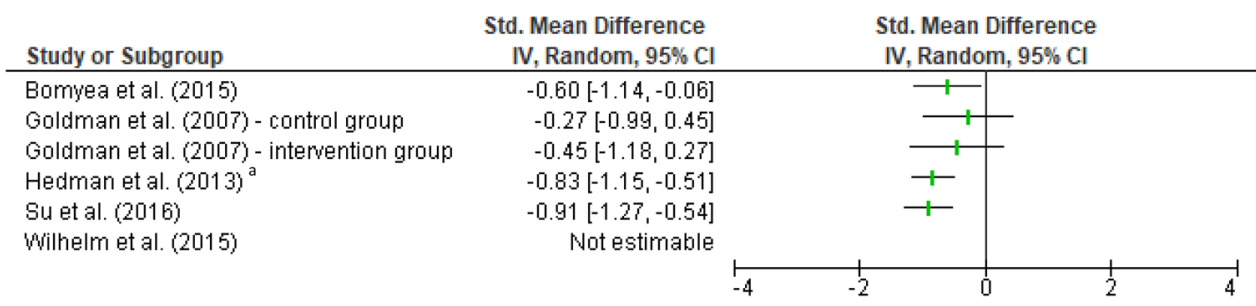

Fig. 3 Forest plot of IU level within individual temporal precedence studies: Magnitude of change in IU level (Hedges' adjusted $g$ ) between first and last assessment point. ${ }^{\mathrm{a}}$ Combined sample effect shown (calculated using intervention and control group means and

bearing on the differing results; although Deschenes and colleagues also controlled pre/post manipulation 'change in irritability' in their analyses, which complicates direct comparison.

Deschenes et al. (2010) provided the only assessment of interpretation bias. The study demonstrated little evidence that IU causes interpretation bias of ambiguous scenarios. There was no significant difference between IU manipulation groups in terms of worry about the scenarios or perceived likelihood of occurrence. Perceived 'badness' of scenarios was also comparable across groups; however, compared to the negative beliefs about uncertainty group, the positive beliefs group did provide higher ratings of the perceived 'goodness' of positive scenario outcomes. Once again, analyses controlled for change in irritability.

Only two studies included mediation analysis exploring relation between experimental group and post-manipulation symptom level (Mosca et al. 2016-studies $1 \& 2$ ). These analyses took the form of exploratory (study 1 ) and confirmatory analyses (study 2). The exploratory analyses found evidence that IU significantly mediated the relationship between manipulation group and worry. However, the reverse model was also significant, suggesting worry may mediate the relationship between manipulation group and IU. The confirmatory analyses conducted (study 2) supported the findings of study one; however, the IU mediator model was reportedly a better fit with the data than the standard deviations). Individual group effects: Intervention group: Hedges' adjusted $g=-1.61[-2.12,-1.11]$; control group: Hedges' adjusted $g=-0.18[-0.62,0.25]$

reverse model. The mediation analyses were limited to one article and one research group. Furthermore, the analyses involved assessment at two time points only (post-manipulation scores, controlling for pre-manipulation scores). Consequently, interpretation of these results should be made with reference to the additional evidence relating to temporal precedence, below.

Intervention Studies Assessing Temporal Precedence Results from the three intervention studies assessing temporal precedence of IU change and anxiety demonstrated some inconsistency-see Table 3. Within the clinical samples, preceding decrease in IU reportedly mediated subsequent decrease in worry across time in those with diagnoses of GAD (Bomyea et al. 2015); and predicted subsequent reduction in health anxiety in those with diagnoses of hypochondriasis (Hedman et al. 2013). However, reverse models (swapping the mediator and outcome) demonstrated inconsistent findings. Hedman et al. (2013) found improvement in health anxiety also predicted a subsequent reduction in IU, whereas Bomyea et al. (2015) did not find preceding worry to mediate change in subsequent IU across time.

In the non-clinical sample reported in Goldman et al. (2007), significant findings were evident but inconsistent across the separate analyses for control and intervention groups. IU was reportedly a significant predictor of subsequent worry and GAD somatic symptoms in the intervention 
group, but not in the control. Conversely, in the control group, preceding worry levels significantly predicted subsequent IU. This reverse relation was not evident in the intervention group. The reported inconsistencies in these findings question their reliability.

\section{Mood, Affect, and Depression}

Experimental Manipulation Studies Investigation of the influence of IU manipulation on emotional state featured in seven studies. The majority of mood and affect assessments included in the experimental manipulation studies utilised VAS. Mosca et al. (2016) reported higher levels of negative affect (NA) in the high compared to low IU group, after controlling for baseline scores, using the PANAS (study 1) and VAS (study 2); no significant difference in positive affect (PA) was found. Similarly, Meeten et al. (2012) found significantly greater increase in sadness in the high compared to low IU group, but no between-group differences in happiness or arousal. Faleer et al. (2017) also supported findings of higher NA in the high compared to low IU group post-manipulation (study 1) but failed to replicate this finding when assessing pre-to-post change in NA (study 2). Although Britton and Davey (2014) also reported significantly higher scores on a general negative mood composite measure in the high compare to low IU group, no significant difference was found on the individual VAS items assessing sadness or negativity. The composite score comprised the sadness and negativity items as well as an anxiety item (previously outlined). The study reported significant betweengroup difference on the anxiety item only, suggesting that the observed effect on the composite score may largely have been driven by anxiety responses rather than overall mood. Finally, Deschenes et al. (2010) found significant decrease in VAS-reported irritability pre/post manipulation in the positive, compared to negative, beliefs about uncertainty group. However, the study authors found no significant betweengroup difference on any other included mood measure.

Consistent with their anxiety analyses, Mosca et al. (2016) conducted exploratory and confirmatory mediational analyses for NA across study one and two. The results were comparable to the anxiety mediation models: IU significantly mediated the relationship between manipulation group and NA; and the reverse model was also significant. Once again, the follow-up confirmatory analyses supported these findings; however, the IU mediator model was reportedly a better fit with the data than the reverse model.

Intervention Studies Assessing Temporal Precedence Only one study investigated the temporal precedence of IU change in relation to depression symptom level (Goldman et al. 2007). Consistent with their findings for worry and GAD symptoms, IU was only a significant predictor of sub- sequent depression level in the intervention group, and not the control group. Conversely, in the control group, preceding depression levels significantly predicted subsequent IU. This reverse relation was not evident in the intervention group.

\section{Obsessions and Compulsions}

Experimental Manipulation Studies Explicit investigation of OCD-relevant constructs within non-clinical samples featured in four studies. These studies provided limited evidence supporting the causal influence of IU. Although one study demonstrated greater perceived threat of intrusive thoughts in the high compared to low IU group (Faleer et al. 2017-study 1), no significant between-group differences were found for inflated responsibility (Britton and Davey 2014), checking behaviour (Faleer et al. 2017-studies 2 \& 3), or threat estimation (Faleer et al. 2017-study 3). Notably, Faleer and colleagues questioned the success of their manipulation in study three.

Intervention Studies Assessing Temporal Precedence The two temporal precedence studies involving individuals with diagnoses of OCD reported contrasting findings. Wilhelm et al. (2015) found that preceding reduction in Perfectionism/IU that was above (compared to at or below) the median magnitude of IU change in the sample, significantly predicted subsequent symptom level of obsessions and compulsions. Su et al. (2016), however, did not find evidence that preceding IU mediated subsequent symptom change across time. Su et al. (2016) also reported the reverse mediation model to be non-significant. Notably, compared to Wilhelm et al. (2015), Su and colleagues did not employ a median split in their analyses, utilised a larger sample size, and controlled for preceding obsession and compulsion symptom level and depression level.

\section{Discussion}

This review evaluated the proposed causal role of IU as a transdiagnostic mechanism of psychological difficulties through synthesis of experimental manipulation and temporal precedence studies. The available evidence was limited to anxiety- and mood-related difficulties (including obsessive-compulsive difficulties). Compared to available correlational evidence (Gentes and Ruscio 2011), the limited quantity and breadth of evidence identified in this review suggests that investigation of the causal influence of IU remains a nascent research area. Whilst the weight of evidence is insufficient to reach absolute conclusions, the findings support the proposition that IU is a mechanism warranting attention and more nuanced consideration. 


\section{Summary of Evidence: The Influence of Intolerance of Uncertainty}

The greatest volume of evidence, and arguably strongest support for IU as a causal mechanism of psychological difficulty, was present for anxiety-related symptoms (excluding obsessive-compulsive difficulties). This finding is predictable given the logical links between fear of the unknown and anxiety, as well as IU's proposed role as a core feature of GAD (Dugas et al. 1998). To an extent, the reviewed experimental manipulation evidence provides some support for the proposition that an increase in IU may affect an increase in symptoms of anxiety, such as worry and catastrophising. However, the evidence is by no means conclusive and caution in interpretation is required due to evident discrepancies between studies. Despite this inconsistency, evaluation of mediation and temporal precedence analyses also provided some evidence that IU change may precede change in anxiety symptoms. Although, the significant reverse models (anxiety symptoms preceding IU) are notable and suggest that bi-directional relationship between IU and anxiety symptoms may also require consideration.

Beyond anxiety, evidence pertaining to affect more broadly appeared less established; although some support for IU's influence upon NA, but not PA, was evident. However, the frequent sole reliance on single-item VAS assessment and demonstration of some inconsistency across studies, limits the overall reliability of this evidence. Evidence relating to temporal precedence analyses was too limited to conclude upon; however, the experimental manipulation mediation analyses and reverse mediation models (Mosca et al. 2016) once again supported the proposition that bi-directional relationships may warrant attention. The relevance of these findings to specific mood-related psychological conditions, such as depression, is debatable. Whilst NA is a component of depression, it is not the sole defining feature of the experience (APA 2013). Where a depression-specific measure was used (i.e., Goldman et al. 2007), results were inconclusive and emphasise the need for further research.

The review considered OCD-related difficulties separately from other anxiety symptoms to reflect the current DSM stratification of conditions (APA 2013). Consistent with anxiety-related difficulties, such as GAD, IU has historically been linked to OCD (OCCWG, 1997). However, despite the established theoretic association, this review found weak evidence supporting IU as a causal mechanism contributing to OCD symptoms. Of the four relevant studies reviewed, only one (Faleer et al. 2017—study 1) demonstrated evidence that IU manipulation influenced a symptom of OCD (i.e., perceived threat of an intrusive thought). Similarly, of the two studies providing analysis of temporal precedence, support for IU change preceding OCD symptom change was only demonstrated in the study involving the smaller sample and employing a categorical split of continuous IU data (Wilhelm et al. 2015), which is arguably less reliable than the comparable evidence presented by Su et al. (2016). Overall, contrary to theoretical predictions, the currently available evidence does not provide strong support for IU as a mechanism of OCD-related difficulties.

Taken as a whole, the evidence reviewed suggests that IU may well exhibit some causal influence over anxiety- and mood-related difficulties and may have the potential to live up to its proposed transdiagnostic mantle (Carleton 2012, 2016). However, the available evidence does not encompass the full spectrum of difficulties proposed to involve IU. For example, no evidence pertaining to symptoms of other psychological difficulties, such as eating disorders (Brown et al. 2017) and psychosis (White and Gumley 2010), was identified through this review. Furthermore, the evidence identified and examined was limited, not only by its diagnostic breadth, but also by its quantity and the level of consistency between findings. These limitations preclude the transdiagnostic mantle being awarded at present. Firm conclusions cannot yet be drawn.

\section{Consideration of Evidential Inconsistencies}

Varying interpretation may be made of the evidential inconsistency reported across both manipulation and temporal precedence studies. First, the evidence may suggest that the influence IU exerts upon psychological difficulties is not as fundamental as previously proposed. Instead, the influence of IU may be more interactional, depending not only on IU but also on the presence of one or more additional variables (e.g., as in Rosen and Knäuper 2009). To the review author's knowledge, there is currently no definitive selection of potential interactive variables. However, existing research suggests moderators may include cognitive (e.g., metacognitive negative beliefs about worry, perceived control over threat; Ruggiero et al. 2012) as well as contextual and behavioural factors (e.g., pervasive technology use becoming a safety behaviour; Carleton et al. 2018). Indeed, Carleton and colleagues' suggestion that the wider context of technology availability and functionality may be contributing to increasing societal levels of IU by facilitating avoidance behaviour emphasises the need to consider systemic, as well as individual-orientated, factors. Second, the differing results may represent a need for a more nuanced conception of IU within experimental and temporal precedence studies. IU may be conceptualised as a composite of the two subcomponents: inhibitory and prospective IU (Carleton et al. 2007; McEvoy and Mahoney 2011). No study in this review evaluated these subcomponents. Some have proposed inhibitory and prospective IU may make differentiable contributions to different psychological difficulties (Mahoney and McEvoy 2012; McEvoy and Mahoney 2011). Consequently, future research 
may obtain results that are more consistent by accounting for these subcomponents. Third, methodological differences between the studies (particularly in terms of differing assessment methods and levels of control) may account for the different findings. Relatedly, only a limited number of the manipulation studies provided evidence of within-group change for high and low IU manipulation groups. Without direct test, evidence of post-manipulation between-group difference, even after demonstrating baseline equivalence, does not guarantee that significant within-group change in each manipulation group is also present. Reliance on postmanipulation group differences to indicate the extent of manipulation is limited and may obscure accurate interpretation. Fourth, the review illustrates a paucity of research providing direct evaluation of causal relationships between IU and psychological difficulties. Compared to the 58 articles included in Gentes and Ruscio's (2011) meta-analysis of correlational evidence, the present review identified only 12 articles. Consequently, one can reasonably argue that the current discrepancies may be manifest of the lack of sufficient research. Resolution of the currently discrepant findings requires a greater volume of high quality research and extension of exploration into other difficulties of proposed relevance, such as eating disorders and psychosis.

\section{Developing Further Research Exploring Causality}

Consideration of the importance of causal evidence warrants additional attention. The most common reason for exclusion of articles from this review during the screening process was that they presented correlational data only. Whilst correlational data provide a useful first step towards identifying relations between variables, these data remain only a first step (Kazdin 2007). Without reliable investigation of whether direct change in a proposed mechanism instigates subsequent change in difficulties, claim of a causal relation is premature. This next step is crucial to gaining a better understanding of the mechanisms of psychological difficulties and the development of mechanism-focused interventions. Whilst we may expect a delay between theoretical proposition of a mechanism and generation of a substantial body of evidence investigating its causal influence, the propositions of IU's relevance to difficulties such as GAD (e.g., Dugas et al. 1998) and OCD (e.g., OCCWG 1997) are well established, yet sufficient evaluation of causality remains lacking. Notably, IU appears considered a clinically relevant target for intervention (see Gillett et al. 2018), despite the limited causal research available to substantiate this focus. This review vehemently echoes Shihata et al. (2016) imploration for a greater body of research explicitly exploring IU as a causal mechanism of psychological difficulty through experimental and longitudinal designs. Recent meta-analytic evidence suggesting that population levels of IU may actually be increasing over time underscores the importance of this call (Carleton et al. 2018).

The criticism suggested here is not peculiar to IU. Mechanisms of psychological difficulties and interventions remain contested and insufficiently understood (Kazdin 2007). If psychological interventions are to take their most effective and efficient form, then the agents of change must be fully understood, and currently they are not. Consequently, let us more frequently move beyond the correlational to gain greater insight into causal influence and the temporal precedence of proposed mechanisms of psychological difficulty and therapeutic change.

\section{Recommendations and Future Directions}

Several recommendations are proposed. First, the discrepant findings warrant additional research assessing causality and temporal precedence to enable reliable conclusions. Inclusion of replications studies would also strengthen continued investigation by providing directly comparable methodologies. Furthermore, a more nuanced consideration of IU, evaluating subcomponents, may prove useful. Second, extending investigation beyond anxiety- and mood-related difficulties to other psychological difficulties in which IU is of proposed relevance (such as eating disorders and psychosis) would help evaluate the full extent of IU's trandiagnostic breadth. Increased involvement of clinical samples would assist this endeavour by investigating difficulties at the extremes of the spectrum of human experience and thus where the potential impact and influence of IU may be most profound. Third, incorporating assessments that provide detailed evaluation, compared to single-item VAS (particularly for mood, affect, and depression), would arguably permit evaluation of multidimensional conceptions of difficulties and avoid unwanted reductionism of complex experiences. Furthermore, as has been suggested elsewhere (see Shihata et al. 2016), attention to measurement validation and consideration of behavioural assessment would improve the reliability of comparison across studies. Finally, bi-directional relationships between IU and symptoms of psychological difficulties may exist. Inclusion of reverse mediation models in temporal precedence studies, and investigation of the effects of symptom manipulation upon IU in experimental designs (e.g., see Britton and Davey 2014, studies 1-2; not eligible for inclusion within this review), may clarify these potential relationships.

\section{Limitations}

There are limitations to this review. First, the evidently nascent area of causal research into IU may necessitate widening consideration to more preliminary research data, such as single-case designs, small scale pilot/clinical 
studies evaluating temporal precedence of change (e.g., Dugas and Ladouceur 2000; Overton and Menzies 2005), and prospective studies with only two assessment points (e.g., Oglesby et al. 2016). The current review excluded these studies to provide a strict threshold for reliability; however, future broader reviews may consider these study types as useful sources of information. Second, only English language and peer-reviewed articles were included. Whilst a pragmatic and common approach within systematic reviews, these criteria limit the breadth of evidence considered. In particular, future reviews may seek to involve translators and/or bilingual researchers to overcome bias towards English language only publications. Third, as stated, the heterogeneity of the study methodology and analyses complicates interpretation of the results. Fourth, the review focused on symptoms of psychological conditions, rather than assessment of full diagnoses, and permitted inclusion of participants from non-clinical populations. Assessment of individual symptoms provides information that is relevant, but not equivalent, to psychiatric diagnoses. Fifth, while IU effect sizes provided were included to inform study comparison, a number of limitations should held in mind. The manipulation studies effect sizes represent post-manipulation group differences only, not change. Temporal precedence effect sizes represent change across two time points only (first and last assessment points) and therefore provide a limited representation of variation across longitudinal assessment and may not capture the greatest level of IU change during the study. Finally, inter-rater agreement demonstrated support for screening reliability, but was limited to a subset of identified articles.

\section{Conclusions}

The present review provides some support for IU as a causal mechanism of anxiety- and mood-related difficulties, but not without substantial caveat. The review results highlight that currently available evidence does not provide an exhaustive account of the role of IU in psychological difficulties and, instead, raises additional questions that require exploration. There is clear need for a greater volume of research, extending beyond correlation to direct investigation of causality, to resolve the evidential discrepancies observed and comprehensively evaluate the proposed breadth of IU's transdiagnostic relevance. Bi-directionality of relations between IU and symptoms of difficulties may also prove a useful avenue for additional consideration. Overall, a more elaborate evidence-base is required to substantiate the proposed extent and utility of IU as a causal, transdiagnostic mechanism underpinning psychological difficulty.
Acknowledgements The review author would like to thank Dr. Miles Thompson for his collaboration on an earlier project from which this research stemmed and Dr. David Moore for providing the additional screening required to enable evaluation of inter-rater reliability.

\section{Compliance with Ethical Standards}

Conflict of interest Ben Rosser declares that he has no conflicts of interest.

Human and Animal Rights This review does not contain any studies with human participants or animals performed by the author himself.

Open Access This article is distributed under the terms of the Creative Commons Attribution 4.0 International License (http://creativeco mmons.org/licenses/by/4.0/), which permits unrestricted use, distribution, and reproduction in any medium, provided you give appropriate credit to the original author(s) and the source, provide a link to the Creative Commons license, and indicate if changes were made.

\section{References}

American Psychiatric Association. (2000). Diagnostic and statistical manual of mental disorders (4th edn.). Washington, DC: American Psychiatric Association.

American Psychiatric Association. (2013). Diagnostic and statistical manual of mental disorders (5th edn.). Washington, DC: American Psychiatric Association.

Awenat, F., Berger, B., Coles, S., Dooley, C., Foster, S., Hanna, J., et al. (2013). Classification of behaviour and experience in relation to functional psychiatric diagnoses: Time for a paradigm shift. Leicester: British Psychological Society.

Boelen, P. A., \& Reijntjes, A. (2009). Intolerance of uncertainty and social anxiety. Journal of Anxiety Disorders, 23, 130-135. https ://doi.org/10.1016/j.janxdis.2008.04.007.

Bomyea, J., Ramsawh, H., Ball, T. M., Taylor, C. T., Paulus, M. P., Lang, A. J., et al. (2015). Intolerance of uncertainty as a mediator of reductions in worry in a cognitive behavioral treatment program for generalized anxiety disorder. Journal of Anxiety Disorders, 33, 90-94. https://doi.org/10.1016/j.janxdis.2015.05.004.

Britton, G. I., \& Davey, G. C. L. (2014). Interrelationships between negative mood and clinical constructs: A motivational systems approach. Frontiers in Psychology, 5, 393-403. https://doi. org/10.3389/fpsyg.2014.00393.

Brown, M., Robinson, L., Campione, G. C., Wuensch, K., Hildebrandt, T., \& Micali, N. (2017). Intolerance of uncertainty in eating disorders: A systematic review and meta-analysis. European Eating Disorders Review, 25, 329-343. https://doi.org/10.1002/erv.2523.

Carleton, R. N. (2012). The intolerance of uncertainty construct in the context of anxiety disorders: Theoretical and practical perspectives. Expert Review of Neurotherapeutics, 12, 937-947. https:// doi.org/10.1586/ERN.12.82.

Carleton, R. N. (2016). Into the unknown: A review and synthesis of contemporary models involving uncertainty. Journal of Anxiety Disorders, 39, 30-43. https://doi.org/10.1016/j.janxd is.2016.02.007.

Carleton, R. N., Desgagné, G., Krakauer, R., \& Hong, R. Y. (2018). Increasing intolerance of uncertainty over time: The potential influence of increasing connectivity. Cognitive Behaviour Therapy. https://doi.org/10.1080/16506073.2018.1476580. 
Carleton, R. N., Duranceau, S., Freeston, M. H., Boelen, P. A., McCabe, R. E., \& Antony, M. M. (2014). "But it might be a heart attack": Intolerance of uncertainty and panic disorder symptoms. Journal of Anxiety Disorders, 28, 463-470. https://doi.org/10.1016/j.janxd is.2014.04.006.

Carleton, R. N., Mulvogue, M. K., Thibodeau, M. A., McCabe, R. E., Antony, M. M., \& Asmundson, G. J. G. (2012). Increasingly certain about uncertainty: Intolerance of uncertainty across anxiety and depression. Journal of Anxiety Disorders, 26, 468-479. https ://doi.org/10.1016/j.janxdis.2012.01.011.

Carleton, R. N., Norton, M. A. P. J., \& Asmundson, G. J. G. (2007). Fearing the unknown: A short version of the intolerance of uncertainty scale. Journal of Anxiety Disorders, 21, 105-117. https:// doi.org/10.1016/j.janxdis.2006.03.014.

Clark, D. A., Purdon, C., \& Byers, E. S. (2000). Appraisal and control of sexual and non-sexual intrusive thoughts in university students. Behaviour Research \& Therapy, 38, 439-455. https://doi. org/10.1016/S0005-7967(99)00047-9.

Craske, M. G., Roy-Byrne, P., Stein, M. B., Sullivan, G., Sherbourne, C., \& Bystritsky, A. (2009). Treatment for anxiety disorders: Efficacy to effectiveness to implementation. Behaviour Research \& Therapy, 47, 931-937. https://doi.org/10.1016/j.brat.2009.07.012.

Cuthbert, B. N., \& Insel, T. R. (2013). Toward the future of psychiatric diagnosis: The seven pillars of RDoC. BMC Medicine, 11, 126-126. https://doi.org/10.1186/1741-7015-11-126.

Davey, G. C. L. (2006). The catastrophic interview procedure. In G. C. L. Davey \& A. Wells (Eds.), Worry and its psychological disorders: Theory, assessment and treatment (pp. 157-178). Chichester: Wiley.

Davey, G. C. L., Hampton, J., Farrell, J., \& Davidson, S. (1992). Some characteristics of worrying: Evidence for worrying and anxiety as separate constructs. Personality \& Individual Differences, 13(2), 133-147. https://doi.org/10.1016/0191-8869(92)90036-O.

Deschenes, S. S., Dugas, M. J., Radomsky, A. S., \& Buhr, K. (2010). Experimental manipulation of beliefs about uncertainty: Effects on interpretive processing and access to threat schemata. Journal of Experimental Psychopathology, 1, 52-70. https://doi. org/10.5127/jep.008510.

Dugas, M. J., Freeston, M. H., Provencher, M. D., Lachance, S., Ladouceur, R., \& Gosselin, P. (2001). Le Questionnaire sur l'Inquiétude et l'Anxiété. Validation dans des échantillons non cliniques et cliniques. [The Worry and Anxiety Questionnaire: Validation in non-clinical and clinical samples.]. Journal de Thérapie Comportementale et Cognitive, 11, 31-36.

Dugas, M. J., Gagnon, F., Ladouceur, R., \& Freeston, M. H. (1998). Generalized anxiety disorder: A preliminary test of a conceptual model. Behaviour Research \& Therapy, 36, 215-226. https://doi. org/10.1016/S0005-7967(97)00070-3.

Dugas, M. J., \& Ladouceur, R. (2000). Treatment of GAD: Targeting intolerance of uncertainty in two types of worry. Behavior Modification, 24, 635-657. https://doi.org/10.1177/0145445500245002.

Einstein, D. A. (2014). Extension of the transdiagnostic model to focus on intolerance of uncertainty: A review of the literature and implications for treatment. Clinical Psychology: Science \& Practice, 21, 280-300. https://doi.org/10.1111/cpsp.12077.

Faleer, H. E., Fergus, T. A., Bailey, B. E., \& Wu, K. D. (2017). Examination of an experimental manipulation of intolerance of uncertainty on obsessive-compulsive outcomes. Journal of Obsessive-Compulsive \& Related Disorders, 15, 64-73. https://doi. org/10.1016/j.jocrd.2017.07.002.

Freeman, D., \& Garety, P. (2014). Advances in understanding and treating persecutory delusions: A review. Social Psychiatry \& Psychiatric Epidemiology, 49, 1179-1189. https://doi.org/10.1007/ s00127-014-0928-7.

Freeston, M. H., Rhéaume, J., Letarte, H., Dugas, M. J., \& Ladouceur, R. (1994). Why do people worry? Personality \&
Individual Differences, 17, 791-802. https://doi.org/10.1016/01918869(94)90048-5.

Garety, P. A., Hemsley, D. R., \& Wessely, S. (1991). Reasoning in deluded schizophrenic and paranoid patients: Biases in performance on a probabilistic inference task. Journal of Nervous \& Mental Disease, 179, 194-201. https://doi.org/10.1097/00005 053-199104000-00003.

Gentes, E. L., \& Ruscio, A. M. (2011). A meta-analysis of the relation of intolerance of uncertainty to symptoms of generalized anxiety disorder, major depressive disorder, and obsessive-compulsive disorder. Clinical Psychology Review, 31, 923-933. https://doi. org/10.1016/j.cpr.2011.05.001.

Gillett, C. B., Bilek, E. L., Hanna, G. L., \& Fitzgerald, K. D. (2018). Intolerance of uncertainty in youth with obsessive-compulsive disorder and generalized anxiety disorder: A transdiagnostic construct with implications for phenomenology and treatment. Clinical Psychology Review, 60, 100-108. https://doi.org/10.1016/j. cpr.2018.01.007.

Goldman, N., Dugas, M. J., Sexton, K. A., \& Gervais, N. J. (2007). The impact of written exposure on worry: A preliminary investigation. Behavior Modification, 31, 512-538. https://doi. org/10.1177/0145445506298651.

Goodman, W. K., Price, L. H., Rasmussen, S. A., Mazure, C., Delgado, P., Heninger, G. R., et al. (1989a). The Yale-Brown obsessive compulsive scale. II. Validity. Archives of General Psychiatry, 46, 1012-1016. https://doi.org/10.1001/archpsyc.1989.01810 110054008.

Goodman, W. K., Price, L. H., Rasmussen, S. A., Mazure, C., Fleischmann, R. L., Hill, C. L., et al. (1989b). The Yale-Brown obsessive compulsive scale. I. Development, use, and reliability. Archives of General Psychiatry, 46, 1006-1011. https://doi. org/10.1001/archpsyc.1989.01810110048007.

Gosselin, P., Ladouceur, R., Evers, A., Laverdiere, A., Routhier, S., \& Tremblay-Picard, M. (2008). Evaluation of intolerance of uncertainty: Development and validation of a new self-report measure. Journal of Anxiety Disorders, 22, 1427-1439. https://doi. org/10.1016/j.janxdis.2008.02.005.

Grenier, S., \& Ladouceur, R. (2004). Manipulation de l'intolérance a l'incertitude et inquiétudes. [Manipulation of Intolerance of Uncertainty and worries.]. Canadian Journal of Behavioural Science, 36, 56-65. https://doi.org/10.1037/h0087216.

Hamilton, M. (1960). A rating scale for depression. Journal of Neurology, Neurosurgery \& Psychiatry, 23, 56-62. https://doi. org/10.1136/jnnp.23.1.56.

Hedman, E., Andersson, E., Andersson, G., Lindefors, N., Lekander, M., Rück, C., et al. (2013). Mediators in internet-based cognitive behavior therapy for severe health anxiety. PLOS ONE, 8, e77752. https://doi.org/10.1371/journal.pone.0077752.

Higgins, J. P. T., \& Green, S. (Eds.) Cochrane Handbook for Systematic Reviews of Interventions Version 5.1.0. The Cochrane collaboration, 2011. Available from http://www.cochrane-handbook.org.

Holaway, R. M., Heimberg, R. G., \& Coles, M. E. (2006). A comparison of intolerance of uncertainty in analogue obsessivecompulsive disorder and generalized anxiety disorder. Journal of Anxiety Disorders, 20, 158-174. https://doi.org/10.1016/j.janxd is.2005.01.002.

Hong, R. Y., \& Cheung, M. W. L. (2015). The structure of cognitive vulnerabilities to depression and anxiety: Evidence for a common core etiologic process based on a meta-analytic review. Clinical Psychological Science, 3, 892-912. https://doi.org/10.1177/21677 02614553789.

Hopko, D. R., Stanley, M. A., Reas, D., Wetherell, J., Beck, J. G., Novy, D., \& Averill, P. M. (2003). Assessing worry in older adults: Confirmatory factor analysis of the Penn State Worry Questionnaire and psychometric properties of an abbreviated 
model. Psychological Assessment, 15, 173-183. https://doi. org/10.1037/1040-3590.15.2.173.

Kazdin, A. E. (2007). Mediators and mechanisms of change in psychotherapy research. Annual Review of Clinical Psychology, 3, 1-27. https://doi.org/10.1146/annurev.clinpsy.3.022806.091432.

Kelly, O. (2009). The escalation of non-pathological worry into generalised anxiety disorder: An investigation of two influential models. Unpublished doctoral dissertation, University of Western Australia, Australia.

Koerner, N., \& Dugas, M. J. (2008). An investigation of appraisals in individuals vulnerable to excessive worry: The role of intolerance of uncertainty. Cognitive Therapy \& Research, 32, 619-638. https ://doi.org/10.1007/s10608-007-9125-2.

Kozak, M. J., \& Foa, E. B. (1997). Mastery of obsessive-compulsive disorder: A cognitive-behavioral approach. San Antonio, TX: The Psychological Corporation.

Krueger, R. F., \& Eaton, N. R. (2015). Transdiagnostic factors of mental disorders. World Psychiatry, 14, 27-29. https://doi. org/10.1002/wps.20175.

Ladouceur, R., Dugas, M. J., Freeston, M. H., Rheaume, J., Blais, F., Boisvert, J. M., et al. (1999). Specificity of generalized anxiety disorder symptoms and processes. Behavior Therapy, 30, 191207. https://doi.org/10.1016/s0005-7894(99)80003-3.

Ladouceur, R., Freeston, M. H., Dumont, J., Letarte, H., Rhéaume, J., Thibodeau, N., \& Gagnon, F. (1992). The Penn State Worry Questionnaire: Psychometric properties of a French translation. Poster presented at the annual convention of the Canadian Psychological Association, Québec, Canada.

Ladouceur, R., Gosselin, P., \& Dugas, M. J. (2000). Experimental manipulation of intolerance of uncertainty: A study of a theoretical model of worry. Behaviour Research \& Therapy, 38, 933-941.

Ladouceur, R., Talbot, F., \& Dugas, M. J. (1997). Behavioral expressions of intolerance of uncertainty in worry. Behavior Modification, 21, 355-371. https://doi.org/10.1177/01454455970213006.

Liberati, A., Altman, D. G., Tetzlaff, J., Mulrow, C., Gøtzsche, P. C., Ioannidis, J. P. A., et al. (2009). The PRISMA statement for reporting systematic reviews and meta-analyses of studies that evaluate healthcare interventions: Explanation and elaboration. BMJ, 339, b2700. https://doi.org/10.1136/bmj.b2700.

MacDonald, B., \& Davey, G. C. (2005). Inflated responsibility and perseverative checking: The effect of negative mood. Journal of Abnormal Psychology, 114, 176-182. https://doi. org/10.1037/0021-843x.114.1.176.

Mahoney, A. E. J., \& McEvoy, P. M. (2012). A transdiagnostic examination of intolerance of uncertatinty across anxiety and depressive disorders. Cognitive Behaviour Therapy, 41, 212-222. https://doi. org/10.1080/16506073.2011.622130.

McEvoy, P. M., \& Mahoney, A. E. J. (2011). Achieving certainty about the structure of intolerance of uncertainty in a treatment-seeking sample with anxiety and depression. Journal of Anxiety Disorders, 25, 112-122. https://doi.org/10.1016/j.janxdis.2010.08.010.

Meeten, F., Dash, S. R., Scarlet, A. L. S., \& Davey, G. C. L. (2012). Investigating the effect of intolerance of uncertainty on catastrophic worrying and mood. Behaviour Research \& Therapy, 50, 690-698. https://doi.org/10.1016/j.brat.2012.08.003.

Meyer, T. J., Miller, M. L., Metzger, R. L., \& Borkovec, T. D. (1990). Development and validation of the Penn state worry questionnaire. Behaviour Research \& Therapy, 28, 487-495. https://doi. org/10.1016/0005-7967(90)90135-6.

Mosca, O., Lauriola, M., \& Carleton, R. N. (2016). Intolerance of uncertainty: A temporary experimental induction procedure. PLoS ONE, 11, 1-15. https://doi.org/10.1371/journal.pone.0155130.

Nolen-Hoeksema, S., \& Watkins, E. R. (2011). A heuristic for developing transdiagnostic models of psychopathology. Perspectives on
Psychological Science, 6, 589-609. https://doi.org/10.1177/17456 91611419672.

Obsessive Compulsive Cognitions Working Group. (1997). Cognitive assessment of obsessive-compulsive disorder. Behaviour Research \& Therapy, 35, 667-681. https://doi.org/10.1016/S0005 -7967(97)00017-X.

Obsessive Compuslive Cognitions Working Group. (2001). Development and initial validation of the obsessive beliefs questionnaire and the interpretation of intrusions inventory. Behaviour Research \& Therapy, 39, 987-1006. https://doi.org/10.1016/ S0005-7967(00)00085-1.

Obsessive Compuslive Cognitions Working Group. (2003). Psychometric validation of the obsessive beliefs questionnaire and the interpretation of intrusions inventory: Part I. Behaviour Research \& Therapy, 41, 863-878. https://doi.org/10.1016/S0005 -7967(02)00099-2.

Obsessive Compuslive Cognitions Working Group. (2005). Psychometric validation of the obsessive belief questionnaire and interpretation of intrusions inventory: Part 2: Factor analyses and testing of a brief version. Behaviour Research \& Therapy, 43, 1527-1542. https://doi.org/10.1016/j.brat.2004.07.010.

Oglesby, M. E., Boffa, J. W., Short, N. A., Raines, A. M., \& Schmidt, N. B. (2016). Intolerance of uncertainty as a predictor of posttraumatic stress symptoms following a traumatic event. Journal of Anxiety Disorders, 41, 82-87.

Overton, S. M., \& Menzies, R. G. (2005). Cognitive change during treatment of compulsive checking. Behaviour Change, 22, 172184. https://doi.org/10.1375/bech.2005.22.3.172.

Radloff, L. S. (1977). The CES-D scale: A self-report depression scale for research in the general population. Applied Psychological Measurement, 1, 385-401. https://doi.org/10.1177/0146621677 00100306

Review Manager (RevMan). (2014). Version 5.3. [Computer program]. Copenhagen: The Nordic Cochrane Centre, The Cochrane Collaboration.

Rosen, N. O., \& Knäuper, B. (2009). A little uncertainty goes a long way: State and trait differences in uncertainty interact to increase information seeking but also increase worry. Health Communication, 24, 228-238. https://doi.org/10.1080/10410230902804125.

Rotge, J. Y., Clair, A. H., Jaafari, N., Hantouche, E. G., Pelissolo, A., Goillandeau, M., et al. (2008). A challenging task for assessment of checking behaviors in obsessive-compulsive disorder. Acta Psychiatrica Scandinavica, 117, 465-473. https://doi.org/10.11 11/j.1600-0447.2008.01173.x.

Ruggiero, G. M., Stapinski, L., Caselli, G., Fiore, F., Gallucci, M., Sassaroli, S., et al. (2012). Beliefs over control and meta-worry interact with the effect of intolerance of uncertainty on worry. Personality \& Individual Differences, 53, 224-230. https://doi. org/10.1016/j.paid.2012.03.016.

Salancik, G. R., \& Conway, M. (1975). Attitude inferences from salient and relevant cognitive content about behavior. Journal of Personality \& Social Psychology, 32, 829-840. https://doi. org/10.1037/0022-3514.32.5.829.

Salkovskis, P. M., Rimes, K. A., Warwick, H. M., \& Clark, D. M. (2002). The health anxiety inventory: Development and validation of scales for the measurement of health anxiety and hypochondriasis. Psychological Medicine, 32, 843-853. https://doi.org/10.1017/ S0033291702005822.

Salkovskis, P. M., Wroe, A. L., Gledhill, A., Morrison, N., Forrester, E., Richards, C., et al. (2000). Responsibility attitudes and interpretations are characteristic of obsessive compulsive disorder. Behaviour Research \& Therapy, 38, 347-372. https://doi. org/10.1016/S0005-7967(99)00071-6.

Shihata, S., McEvoy, P., Mullan, B. A., \& Carleton, R. N. (2016). Intolerance of uncertainty in emotional disorders: What uncertainties 
remain? Journal of Anxiety Disorders, 41, 115-124. https://doi. org/10.1016/j.janxdis.2016.05.001.

Shihata, S., McEvoy, P. M., \& Mullan, B. A. (2017). Pathways from uncertainty to anxiety: An evaluation of a hierarchical model of trait and disorder-specific intolerance of uncertainty on anxiety disorder symptoms. Journal of Anxiety Disorders, 45, 72-79. https ://doi.org/10.1016/j.janxdis.2016.12.001.

Spielberger, C. D. (1983). State-trait anxiety inventory for adults. Palo Alto: Mind Garden.

Stöber, J., \& Bittencourt, J. (1998). Weekly assessment of worry: An adaptation of the Penn State Worry Questionnaire for monitoring changes during treatment. Behaviour Research \& Therapy, 36, 645-656. https://doi.org/10.1016/S0005-7967(98)00031-X.

Su, Y. J., Carpenter, J. K., Zandberg, L. J., Simpson, H. B., \& Foa, E. B. (2016). Cognitive mediation of symptom change in exposure and response prevention for obsessive-compulsive disorder. Behavior Therapy, 47, 474-486. https://doi.org/10.1016/j.beth.2016.03.003.

Tolin, D. F., Abramowitz, J. S., Brigidi, B. D., \& Foa, E. B. (2003). Intolerance of uncertainty in obsessive-compulsive disorder. Journal of Anxiety Disorders, 17, 233-242. https://doi.org/10.1016/ S0887-6185(02)00182-2.

Vasey, M. W., \& Borkovec, T. D. (1992). A catastrophizing assessment of worrisome thoughts. Cognitive Therapy \& Research, 16, 505-520. https://doi.org/10.1007/bf01175138.

Watson, D., Clark, L. A., \& Tellegen, A. (1988). Development and validation of brief measures of positive and negative affect: The PANAS scales. Journal of Personality \& Social Psychology, 54, 1063-1070. https://doi.org/10.1037/0022-3514.54.6.1063.
Webster, D. M., \& Kruglanski, A. W. (1994). Individual differences in need for cognitive closure. Journal of Personality \& Social Psychology, 67, 1049-1062. https://doi. org/10.1037/0022-3514.67.6.1049.

White, R. G., \& Gumley, A. (2010). Intolerance of uncertainty and distress associated with the experience of psychosis. Psychology \& Psychotherapy: Theory, Research \& Practice, 83, 317-324. https://doi.org/10.1348/147608309X477572.

Widiger, T. A., \& Samuel, D. B. (2005). Diagnostic categories or dimensions? A question for the diagnostic and statistical manual of mental disorders-fifth edition. Journal of Abnormal Psychology, 114, 494-504. https://doi.org/10.1037/0021-843x.114.4.494.

Wilhelm, S., Berman, N. C., Keshaviah, A., Schwartz, R. A., \& Steketee, G. (2015). Mechanisms of change in cognitive therapy for obsessive compulsive disorder: Role of maladaptive beliefs and schemas. Behaviour Research \& Therapy, 65, 5-10. https:// doi.org/10.1016/j.brat.2014.12.006.

Wilhelm, S., Steketee, G., Fama, J. M., Buhlmann, U., Teachman, B. A., \& Golan, E. (2009). Modular cognitive therapy for obsessivecompulsive disorder: A waitlist controlled trial. Journal of Cognitive Psychotherapy, 23(4), 294-305.

Wilhelm, S., Steketee, G., Reilly-Harrington, N. A., Deckersbach, T., Buhlmann, U., \& Baer, L. (2005). Effectiveness of cognitive therapy for obsessive-compulsive disorder: An open trial. Journal of Cognitive Psychotherapy, 19, 173-179. https://doi.org/10.1891/ jcop.19.2.173.66792 